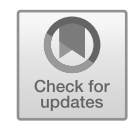

\title{
5 Erprobung einer ARqE-Anwendung
}

Forschungsfragen zielen aus ARqE-Perspektive nicht auf "principles of psychological functioning that are abstract, general, and universal" (Sampson, 1978, S. 1333; vgl. Eingangszitate dieser Arbeit auf S. 1). Stattdessen werden Konzepte des menschlichen Erlebens, Denkens, Fühlens und Handelns als einzelne Manifestationen von Möglichkeiten verstanden. Diese Manifestationen sind situativ real, sie haben sich realisiert. Je nach Gegenstand mag auch materialisiert das passendere Wort sein. Die spezifischen Manifestationen wiederum bedingen Konsequenzen mit und sind dadurch selbst wirksam. Gerade im Bereich des menschlichen Erlebens können diese Manifestationen situiert hochrelevante Ereignisse darstellen. Wir begegnen lokalen und temporären Fakten der Welt, weil die relations der relata aus mehreren Möglichkeiten intra-aktiv die vorhandene dann-Realisierung (per agential cut) herausgeschnitten haben. Oft genug sind auch wir Menschen - und gerade Forschende - diejenigen, die die agential cuts setzen. Forschende stellen mit ARqE-Grundannahmen also andere Fragen, als wenn sie davon ausgingen, universale Fakten zu suchen. Wir können verschiedene Fragen stellen: Was ist wo für wen eine Realisierung, die nicht erst Forschende herstellen, indem sie danach fragen. Welche weiteren Realisierungen wären möglich, d. h., wie kann die Welt noch aussehen? Welche Intra-aktionen begünstigen welche Manifestation(en)? Welche Herstellungen können Menschen mitgestalten und welche (noch) nicht? Mit ARqE-Grundannahmen fragen Forschende nach Möglichkeitsraum; falls sie nach ontologischen Gegebenheiten fragen, dann nur lokal, temporär und bezogen auf bestimmte Menschen (d. h., bezogen auf situierte ontoepistemologische Gegebenheiten).

Solche Analysen sind schon über die unterschiedlichsten Disziplinen hinaus vorhanden, wenn auch nicht unter dem Label des Agential Realism. Beispielsweise sehe ich Foucaults (1968) Beschreibung, wie sich Geisteskrankheit in den heutigen europäisch geprägten Gebieten realisieren konnte, als Darstellung von wirkenden agential cuts. Er fragt unter anderem: „Liegt nicht in der Krankheit ein ganzer Knoten von Bedeutungen, der aus dem Umkreis, in dem sie aufgetreten ist, herstammt - und zuvörderst die einfache Tatsache, daß sie in diesem Umkreis als Krankheit abgegrenzt wird?“ (Foucault, 1968, S. 90) Er

(C) Der/die Autor(en) 2018

J. Scholz, Agential Realism als Basis queer(end)er

Experimentalpsychologie, https://doi.org/10.1007/978-3-658-22644-2_5 
kann sehr detailliert nachzeichnen, welche Interpretationen und Sichtweisen welchen Verhaltens in welchen Gesellschaften zu welcher Zeit erst zur Gestalt Geisteskrankheit führten. An diesen wirkenden cuts haben sich und beteiligen sich bis heute auch Forschende, zum Beispiel durch Klassifikation von sogenannten Störungen in einem ICD (International Classification of Diseases). Auch wurden schon Auswirkungen von bestimmten Manifestationen auf andere Manifestationen beschrieben, ohne diese jeweils essentialisierend zu behandeln. Butler arbeitete eine heterosexuelle Matrix und Foucault ein Sexualitätsdispositiv als zeit- und kontextgebundenes relatum-within-relations heraus und beide können Folge-Intra-aktionen auf andere zeit- und kontextgebundene relata, z. B. geschlechtliche Kategorisierung, beschreiben. Solche Analysen können und sollten aus AR-Perspektive zusätzlich Konfigurationen einbeziehen, die traditionell als physisch oder materiell - und damit als nicht-kulturell - galten. Um eine Forschungsanstrengung agential realist $\mathrm{zu}$ nennen, ist ist es $\mathrm{m}$. E. jedoch nicht notwendig, dass materielle Konfigurationen eine unmittelbare Rolle spielen. Das schlussfolgere ich daraus, dass Barad zwar immer von material-discoursive als wirkenden Konfigurationen ausgeht, jedoch gerade nicht unterscheiden will, dass die einen nur materielle Bedingungen seien und die anderen diskursive.

Ich behaupte also, dass auch solche Analysen empirisch durchgeführt werden können, die ihre mit dem empirischem Vorgehen erzeugten Ergebnisse nicht so behandeln, als wären es "human-independent facts about nature" (Barad, 1996, S. 162). Dann kann damit gearbeitet werden, dass Wissenschaft funktioniert (vgl. Barad, 1996, S. 162) - solange dies verantwortlich geschieht (vgl. Kapitel 4).

Die Durchführung von konkreten Studien wirft zusätzliche Probleme auf, für die es zahlreiche raffinierte Umsetzungsmöglichkeiten zu entwickeln gilt. Deren zukünftige Lösung erscheint mir jedoch außerordentlich erstrebenswert. In diesem Kapitel will ich den Versuch, einen Effekt zu realisieren, an einem Beispiel diskutieren. Der Ausdruck des Realisierungsversuches soll hervorheben, dass wir mit Empirie nicht beweisen, wie etwas in der Welt ontologisch ist, sondern höchstens eine Realisierung mit möglichst vielen ihrer mitbedingenden Konfigurationen vorführen. Die schrittweise Besprechung einer 
Studie, die an ein traditionelles Muster von sozialpsychologischen Studien angelehnt ist, und ihrer methodischen Herausforderungen, soll erste Lösungen und offene Problemstellen demonstrieren. Durch die Vielzahl der Möglichkeiten, wie eine Forschungsfrage gestellt werden kann, ist sicher nur ein Teil hiervon auf andere Studien übertragbar. Die Darstellung hier dient der ersten Veranschaulichung und nicht der konkreten Anleitung.

Ein erstes Problem nenne ich, dass ich auf wenige explizite experimentalpsychologische Einsichten über wirkende Intra-aktionen, agential cuts und relations der relata zurückgreifen kann. Dies führe ich darauf zurück, dass derzeit bei den meisten (psychologischen) Studien unsicher ist, wie stark die Messung an der Phänomengestalt mitwirkt. Mit mehr Zugriff auf Forschung aus ARqE-Perspektive sollten sich konkretere Einschätzungen beschreiben lassen. Dennoch kann meines Erachtens auf Studien referenziert werden, die nicht unter einer ARqE-Perspektive durchgeführt wurden, sofern der konkrete Zusammenhang, mit dem argumentiert wird, der agential realist Logik nicht widerspricht. So kann ein Zusammenhang zwischen zwei Realisierungen bzw. relata-withinrelations auch dann anerkannt werden, wenn bestimmte Aussagen in ARqE anders formuliert oder Autor_inn_en aus ARqE-Perspektive gewisse Schlüsse anders ziehen würden. Als Beispiel soll folgender Schluss aus klassisch experimenteller Perspektive dienen: "[C]hildren who reported strong felt pressure for gender differentiation yet saw themselves as gender atypical, were discontent with their gender, or lacked self-esteem also became more aggressive toward gender-nonconforming peers" (Pauletti, Cooper \& Perry, 2014, S. 860). Dieser müsste aus ARqE-Perspektive so spezifiziert werden: "Children who reported strong felt pressure for gender differentiation yet indicated to see themselves as gender atypical, indicated that they were discontent with their gender, or reported to lack self-esteem (...)". Damit würde offen gelassen, ob nicht erst die spezifische Art der Messung von gender atypicality eben jene erst hergestellt hat. Die Beschreibung des Effektes - dass der Selbstbericht von jener Atypikalität (und sei es auf bzw. durch Nachfrage) mit bestimmten so-und-so verstandenen Aggressionen zusammenhängt (und dies ggf. nicht die einzige Realisierungsmöglichkeit ist) - könnte danach jedoch argumentativ verwendet werden. Je nach Verwendungswunsch der Beschreibung ist die darin enthaltene 
Heternormativität der Zweigeschlechtlichkeit, das wahrscheinlich fehlende Intersektionaldenken und die örtliche und zeitliche Einschränkung einer solchen Aussage mitzutransportieren. Man muss also die Annahme der Stabilität von Geschlechtsidentität nicht teilen, um anerkennen zu können, dass lokal und situativ ein Antwortverhalten X (einer bestimmten Gruppe) mit einem so-und-so beschriebenen Verhalten Y zusamenhängen kann. Verständlicherweise nimmt eine solche situierte Beschreibung den Universalismus aus einem Argument was gerade eines der ARqE-Ziele ist.

Im weiteren Verlauf werden einige praktische Empfehlungen für eine empirische Effekt-Realisierung aus ARqE-Perspektive wiederholt. Dafür wird im Text abgewechselt zwischen der Besprechung von Formulierungen und den konkreten Formulierungen selbst. Zur visuellen Verdeutlichung der Wechsel ist der Text, der eine konkrete Auseinandersetzung aus ARqE Perspektive darstellen soll, gegenüber der Besprechung der Formulierungen eingerückt und in einer anderen Schriftart.

\subsection{Auswahl - mit Begründung - des zu untersuchenden Phänomens}

Bei der Auswahl eines zu untersuchenden Phänomens können wir aus der ARqE-Perspektive nicht davon ausgehen, dass wir versuchen zu entdecken, was schon gegeben ist. Vielmehr wissen wir zunächst nicht, ob ein Phänomen ohne unsere Forschungstätigkeit unbestimmt ist oder dieses durch andere Intraaktionen (die nicht Teil unser spezifisches Eingreifen sind) eine Realisierung in der gleichen Zeit und am gleichen Ort erfährt. Je nach Phänomen müssen wir entweder damit rechnen, dass wir a) die Manifestation durch unsere Forschung erst herstellen, oder dass wir b) etwas untersuchen können, was durch andere Intra-aktionen als die unserer Forschung hergestellt wird (wir aber möglicherweise mit einer Untersuchung dennoch an der Wiederholung und Aufrechterhaltung beteiligt wären). Eine Mischform von a) und b) wäre, wenn z. B. kulturelle Intra-aktionen eine Manifestation auch ohne unsere Forschung realisieren, unsere 
Forschung aber eine spezifische Dimension herstellt (z. B. eine Skala von 1 bis 10 mit Bezeichnung), auf der diese Manifestation dann neu mit-hergestellt wird. Wenn wir uns beispielsweise für geschlechtliche Selbstkategorisierung interessieren, kann die Begründung in der ARqE nicht lauten, dass wir einfach zu wenig darüber wüssten (z. B. im Sinne einer Tatsache der zwangsläufigen, unvermeidbaren geschlechtlichen Selbstkategorisierung). Stattdessen müssten wir in ARqE-Studien zunächst davon ausgehen, dass die geschlechtliche Selbstkategorisierung unbestimmt und nicht per se gegeben ist, und begründen, warum wir dieses Konzept herstellen oder seine Herstellung wiederholen. Selbst wenn wir davon ausgingen (etwa durch vorangegangene ARqE-Forschung), dass viele andere Intra-aktionen zur Herstellung des Phänomens beitragen, muss aus ARqE-Perspektive eine wissenschaftliche Aus-einandersetzung erklären, warum die Reifikation des Konzeptes eingegangen wird. Damit wird die Studie schon an diesem Punkt angreifbar und erfüllt einen Teil der besonderen Verantwortung von Forschenden für die Gestalt ihres Ergebnisses (siehe Kapitel 4.2.5). Eine konkrete Formulierung könnte wie folgt lauten:

Unter Anwendung der Perspektive der Agential Realist queer(end)en Experimentalpsychologie (ARqE) fragt die folgende Auseinandersetzung nach möglichen Realisierungen eines Phänomens und erprobt hierfür potentiell cut-herstellende Intra-aktionen.

Diese Art von übergeordneter, sehr unkonkreter Beschreibung, was in der Studie kommen wird, macht in dieser Arbeit Sinn, da sie erst an das prinzipielle Vorgehen heranführt. Eine alleinstehende Studie aus ARqE-Perspektive sollte direkt mit konkreten Inhalten beginnen.

Konkret wähle ich das Phänomen der geschlechtlichen Selbstkategorisierung und es werden Konfigurationen zur verschiedentlichen Herstellung derselben ausprobiert.

Ganz bewusst wird nicht die Idee der Veränderung eingesetzt, so als ob gewisse Konfigurationen eine geschlechtliche Selbstkategorisierung verändern würden. Vielmehr wird erprobt, welche Intra-aktionen offensichtlich einen Anteil an einer Realisierungsform haben. 
Weiterhin wird eine Hypothese aus der deterministischen Psychologie aufgenommen und versucht, ob sich deren vorhergesagtes Phänomen realisieren lässt.

Je nach Veröffentlichungsform kann problemlos das Konzept eines sog. Abstracts im Sinne einer einführenden Kurzzusammenfassung für die ARqE übernommen werden. Darin würden konkret die zusammenfassenden Aussagen der Auseinandersetzung genannt werden. In der vorliegenden Arbeit habe ich kein Abstract realisiert, sondern fahre mit einem in das Thema einführenden Abschnitt fort:

Ich gehe hierin davon aus, dass aktuell in europäisch und US-amerikanisch geprägten Gesellschaften verschiedenste Vorgänge (im Sinne von cutherstellenden Konfigurationen) Menschen häufig zu einer geschlechtlichen Selbstkategorisierung anregen (bishin diese zu forcieren). Die Selbstkategorisierung findet in privaten Situationen statt, in denen Menschen als Frau z. B. andere Schönheitshandlungen vollziehen als als Mann. Zudem „findet in allen gesellschaftlichen Bereichen permanent eine entsprechende Einordnung und Abfrage statt (Personalausweis, Krankenakte, Versicherungsvertrag etc.)“ (Döring, 2013, S. 97) - bis hin zur wissenschaftlichen Nachfrage nach dem Geschlecht der Studienpartner_innen: „Kein Fragebogen ohne Geschlechtsabfrage - das ist die Forschungsrealität in den Sozialwissenschaften." (Döring, 2013, S. 94) Gleichzeitig erachtet meine Auseinandersetzung Geschlecht (neben ethnischer Zugehörigkeit und sozioökonomischer Klasse) als eine der drei zentralen Strukturierungskategorien von heutigen, europäisch und US-amerikanisch geprägten Gesellschaften, was die geschlechtliche (Selbst- und Fremd-) Kategorisierung im Moment noch zu einem sozial äußert relevanten Vorgang macht. Die Einordnung kann für einzelne Menschen persönlich irrelevant sein, aber kulturell erscheint sie mir derzeit noch hochrelevant zu sein. Mit der aktuellen und örtlichen Häufigkeit sowie der Relevanz der geschlechtlichen Selbstkategorisierung begründe ich, warum ich hier die Herstellung des Phänomens wiederhole, obwohl dies problematische Konsequenzen mit sich bringt. Beispielhaft möchte ich eine solche Konsequenz andeuten: Die 
Annahmen, wie Frauen und Männer (weitere Geschlechter werden in der Regel nicht genannt) typischerweise sind, sind in den genannten Kulturen nicht nur deskriptiv, sondern haben auch präskriptive Funktion (Prentice \& Carranza, 2002). Das bedeutet, dass eine geschlechtliche Kategorisierung eine Art kultureller Vorschriften mit sich bringt, wie beispielsweise eine als Frau kategorisierte Person zu sein hat, um nicht soziale Sanktionen zu riskieren (vgl. Prentice \& Carranza, 2002). Einige Forschungsarbeiten zu solchen Sanktionen tragen Pauletti et al. (2014) zusammen. Die vorliegende Studie birgt also u. a. die Gefahr, durch die Reifikation des Phänomens geschlechtliche Selbstkategorisierung an der Herstellung von geschlechtsstereotypen Vorschriften beteiligt zu sein.

Hier wird also angenommen, dass heutzutage in den europäisch und US-amerikanisch geprägten Gesellschaften zahlreiche Intra-aktionen häufig zu einer geschlechtlichen Selbstkategorisierung führen. Die problematischen Seiten dessen kann eine_n Forscher_in dazu veranlassen, dieses Konzept gerade nicht $\mathrm{zu}$ wiederholen, oder aber dazu, etwas queertheoretisch Kritisches zur Konzeptionalisierung beizutragen. Hier wird Letzteres angestrebt und gleichzeitig versucht, Sensibilität für die problematischen Konsequenzen des Vorgehens zu zeigen.

Manche Theoretiker_inne_n gehen von folgender Prämisse aus: “[G]ender identity in its strictest sense (i.e., perceiving oneself as a boy or a girl) develops during the early childhood period" (Clemans, DeRose, Graber \& Brooks-Gunn, 2010, S. 529). Weiter wird häufig davon ausgegangen, dass diese gender identity dann normalerweise bestehen bleibt. Von dieser Vorstellung abweichend gehe ich aus queertheoretischer Perspektive davon aus, dass geschlechtliche Identität nicht dauerhaft inhärent in Menschen manifestiert ist. Vielmehr nehme ich entsprechend des doing-genderAnsatzes (vgl. West \& Zimmerman, 1987; Kessler \& McKenna, 1978, 2006) an, dass Geschlecht etwas ist, das wir tun und das situativ neu hergestellt wird, und nicht etwas, das wir haben. Eine Selbstkategorisierung wird demnach erst nach einer Abfrage von geschlechtlicher Identität vorgenommen. Abfrage muss dabei keine von jemand intendierte direkte verba- 
le Frage sein, sondern kann auch die Salient-Werdung einer kulturell angenommenen Geschlechtskategorie in einer Situation durch spezifische Auslöser meinen. Gemäß den ARqE-Aussagen gehe ich also davon aus, dass geschlechtliche Selbstkategorisierung solange nicht existiert (unbestimmt ist), bis sie durch entsprechende Intra-aktionen hervorgerufen wird, und nur durch die wiederholte Herstellung stabil erscheinen mag.

Solche Formulierungen sollen deutlich benennen, dass ein Vorkommnis in der Welt (hier: geschlechtliche Selbstkategorisierung) nicht per se gegeben ist, auch wenn es häufig anzutreffen sein mag. Zwei Komponenten (Häufigkeit und Relevanz) werden als Gründe für die Wiederholung genannt, während die Problematik der Wiederholung - aufgrund derer man sich in anderen Fällen gegen die Wiederholung dieses Phänomens entscheiden mag - ebenfalls genannt wird. Durch die Verwendung von ,aktuelle und örtliche Häufigkeit“ (S. 200) soll außerdem deutlich gemacht weden, dass Häufigkeit und Relevanz sehr temporär und lokal beschränkte Gegebenheiten sind.

Mit den hier erprobten Formulierungen zur Begründung der Wahl eines Phänomens, wird versucht, die Vorannahmen zu explizieren, um den Verständnisrahmen der Zusammenhänge, die erprobt werden sollen, und die daraus folgenden Interpretationen, deutlich $\mathrm{zu}$ machen und um sie der Kritik zu exponieren. Sie sollen außerdem betonen, dass die in der Studie beschriebenen Zusammenhänge höchstwahrscheinlich nicht die einzigen Realisierungsmöglichkeiten darstellen. Ähnliches wird auch bei der Formulierung der Forschungsfrage bzw. der konkreten Hypothesen angestebt.

\subsection{Auswahl - mit Begründung - der Forschungsfrage und Methode}

Mit der Begründung der Wahl des zu untersuchenden Phänomens und seiner Konzeptionalisierung hängt die Formulierung der Forschungsfrage zusammen. Auch hier muss in ARqE erklärt werden, warum die Frage so gestellt wird und nicht anders. Feministischen Theorien weisen darauf hin, dass es eine 
Wirkung hat, wenn das Erleben und Handeln von Frauen besonders erklärt wird, und damit das Verhalten von Männern als die normale Variante erscheinen lässt (siehe z. B. Wilkinson, 1996). Gleichermaßen weist die LGBT Psychology darauf hin, dass es eine Wirkung hat, wenn das Erleben und Handeln von Homosexuellen besonders erklärt wird, und damit das Verhalten von Heterosexuellen als die normale Variante erscheinen lässt (siehe beispielsweise Clarke, Ellis, Peel \& Riggs, 2010). Das Phänomen hat Einzug in die akademische Psychologie gehalten (siehe u. a. Miller, Taylor \& Buck, 1991; Bruckmüller, 2013). Bruckmüller, Hegarty und Abele (2012) können genau dies empirisch vorführen: Lokal kann es eine Wirkung haben, was in einer Formulierung als linguistische Norm verwendet wird und was als der zu erklärende Faktor gilt. Aus ARqE-Perspektive ist eine solche Wirkung dann eine mögliche Realisierung, die sich unter den installierten Konfigurationen matrialisierte.

Wie die Güte von Formulierungsweisen für Begründungen von Forschungsfragen aus ARqE-Perspektive beurteilt werden kann, geht über den Rahmen dieser Arbeit hinaus. Ich rege hierzu explizit Arbeiten an. Für den Anfang kann es hilfreich sein, stets die Frage zu bearbeiten, warum das untersuchte Objekt so und nicht anders konzeptioniert wird bzw. warum die Forschungsfrage so und nicht anders gestellt wird.

Hier soll erprobt werden, ob eine direkte verbale Abfrage von Geschlecht eine der Intra-aktionen sein kann, die bei der Herstellung einer geschlechtlichen Selbstkategorisierung mitwirken. Dann wäre die Art und Weise der Messung dieser Selbstkategorisierung, also die Gestalt der verbalen Abfrage, ein das-Ergebnis-mitbestimmender Apparat im Sinne von Barad (2007).

Die Hypothesentestung kann hier fast analog zur klassichen Experimentalpsychologie ablaufen, mit dem Unterschied, dass sich die Hypothese auf die Realisierung einer Möglichkeit bezieht - es wird also getestet, was situativ möglich ist, und nicht, wie etwas per se beschaffen sei (vgl. Kap. 4).

Traditionellerweise wird Geschlecht in sozialwissenschaftlichen Fragebögen binär abgefragt (Döring, 2013). Studienpartner_innen sollen also auf 
die Frage nach ihrem Geschlecht entweder die Antwort „weiblich“ oder „männlich“ ankreuzen. Formuliert man diese Frage und vor allem ihre Antwortmöglichkeiten nonbinär, dann könnte diese Frageart im Sinne der ARqE eine Konfigurationskonstellation des Ergebnis-mit-herstellenden Messapparates darstellen. Die Wirkung einer solchen Konfigurationskonstellation soll hier empirisch erprobt werden. Es soll geprüft werden, ob eine Anders-Formulierung einer klassischen Frage eine andere als die klassische Realisierung erzeugen kann. Die erste Hypothese lautet daher: Wird die Geschlechtsabfrage in einem Fragebogen nonbinär gestellt, so stellt diese Frage eine größere Geschlechtervielfalt in den Antworten her, als wenn die Geschlechtsabfrage klassisch binär gestellt wird.

Selbstredend kann ein solches Experiment nicht offenbaren, ob nun die klassische Annahme einer inhärenten oder die agential realist queer-Annahme einer ständig herzustellenden Geschlechtsidentität der Wahrheit entspräche wie es Ziel des klassischen Realismus‘ wäre (vgl. Kap. 3.1.2). Wohl kann eine solche Studie aber - was Ziel des Agential Realism ist - offenbaren, ob eine Herstellung möglich ist. Herstellung beschränkt sich hier auf Antworten in einem Fragebogen und es ist nicht etwa die Rede von einem "own sense of themselves as a boy/man or as a girl/woman" (Smith, Johnston-Robledo, McHugh \& Chrisler, 2010, S. 364) oder als etwas anderes (außer boy/man bzw. girl/woman) oder als nichts. So werden die Ergebnisse desselben Experimentes aus einer klassisch realistischen Perspektive anders interpretiert als aus ARqE-Perspektive. Im traditionellen Verständnis würden hier Treatment (also die experimentelle Bedingungsvariation) und Messung in einem Punkt zusammenfallen. Wenn zwei verschiedene Arten der Geschlechtsabfrage unterschiedliche Ergebnisse produzieren, so wäre aus klassisch experimentalpsychologischer Sicht mindestens eine nicht als Messinstrument geeignet (bzw. würde auf einem zweiten Faktor laden). Döring (2013) schreibt, dass „eine Befragung nicht nur eine Datenerhebung, sondern automatisch auch eine Intervention dahingehend darstellt, dass die Art der Fragen die Befragten zum Nach- und ggf. auch Umdenken animieren kann (sogenannte instrumentelle Reaktivität)“ (Döring, 2013, S. 103). Allerdings wird diese instrumentelle Reaktivität klassischerweise als Störquelle erachtet und nicht als Selbstverständlichkeit unserer psychologischen Forschung (und aller 
Intra-aktionen). Demgegenüber wird hier entlang der ARqE davon ausgegangen, dass eine Messung immer ihren Teil zur Phänomenherstellung beiträgt. Es könnte für zukünftige Forschungen interessant sein, wie groß dieser Teil jeweils ist, weil daraus unterschiedliche Interpretationen abgeleitet würden. So mag der Anteil situativ auch vernachlässigbar sein, aber davon können wir nicht per se ausgehen.

Dass die Art und Weise der Messung ein das-Ergebnismitbestimmender Apparat ist (vgl. S. 203), müsste in Arbeiten aus ARqEPerspektive nicht explizit benannt werden, da dies inhärentes Verständnis der ARqE-Perspektive ist. Hier wird - abweichend davon - dem Umstand Rechnung getragen, dass diese Perspektive im Moment erst vorgestellt wird.

Ich vergleiche also die Antworten einer Gruppe von Studienpartner_innen auf die klassisch binär formulierte Frage mit den Antworten einer Gruppe auf eine nonbinär formulierte Frage. Damit jedoch nicht eine Gruppe der Studienpartner_innen mit einer rein heteronormativen und unproblematisierten geschlechtlichen Selbstkategorisierungsaufgabe (binäre Abfrage) konfrontiert wird, werden beide Arten der Geschlechtsabfrage (binär und nonbinär) allen Studienpartner_innen vorgelegt. Jede_r Studienpartner_in beantwortet also zweimal eine geschlechtliche Selbstkategorisierung.

Es ist klassisch experimentalpsychologisch wohl eher unüblich, ethische Überlegungen solcher Art (womit manche Studienpartner_innen nicht konfrontiert werden sollen) das Design der Studie mitbestimmen zu lassen. Solch reflexives Vorgehen sollte jedoch aus Perspektive der ARqE zum Standard werden.

Das zweimalige Antworten wird vermutlich die Beschaffenheit der Konstrukte mitbestimmen. Beispielsweise legen aktuelle Konversationsnormen (siehe Wänke, 2007) des europäisch und US-amerikanisch geprägten Raumes nahe, dass eine vernünftig fragende Person/Studie nicht zweimal das (vermeintlich) Selbe fragt. Es ist möglich, den Fragebogen so zu gestalten, dass bei der ersten Abfrage noch nicht ersichtlich ist, dass die gleiche Frage 
noch einmal gestellt wird. Spätestens bei der zweiten Abfrage legen die Konversationsnormen den Studienpartner_inne_n jedoch nahe, dass diesmal etwas anderes als zuvor gefragt würde. Es kann also eine Rolle spielen, welche Frageart zuerst und welche als zweites präsentiert wird. Daher nehme ich an, dass auch die Reihenfolge, in der die verschiedenen Fragearten (binär und nonbinär) eingesetzt werden, ein Ergebnismitbestimmender Teil der Konfiguration ist. Hier wird die These des Effektes der Reihenfolge zunächst ungerichtet erprobt.

Wohlgemerkt wird nicht getestet, ob hier wirklich Konversationsnormen den agential cut mit-herstellen, denn dies müsste anders vorgeführt werden. Zunächst wird erprobt, ob die Reihenfolge eine mitbestimmende Konfiguration ist. Dann könnte weiter gefragt werden, welche Konfigurationen genau die mitbeteiligten Intra-aktionen stellen.

Aus Perspektive der ARqE wäre das Ergebnis, dass die Reihenfolge die Beschaffenheit der Antwort mitgestaltet, explizit keine Verzerrung von einer als korrekt angenommenen Antwort, sondern gehörte zum Phänomen der geschlechtlichen Selbstkategorisierung dazu. Entsprechend wird hier von Barad'schen Intra-aktionen gesprochen und nicht von Verzerrungen. Wenn die Hypothese formuliert ist, folgt in einer klassischen Studie die Operationalisierung der Konstrukte. Wie in 3.2.3. beschrieben, gibt es in $\mathrm{ARqE}$ keine Operationalisierung, weil nicht von zu messenden Eigenschaften ausgegangen wird, für die möglichst gute Indikatoren gefunden werden müssten. Deshalb folgt nun einfach die Spezifizierung der Konfigurationen.

In dieser Studie wird versucht, durch folgende Konfigurationen des Messapparates das Ergebnis der geschlechtlichen Selbstkategorisierung mitzubestimmen: erstens, durch die unterschiedliche Formulierung von Frage und Antwortmöglichkeit, und zweitens, durch die Reihenfolge der Abfrage. Dieser Aufbau erlaubt außerdem das unkomplizierte Ausprobieren einer weiteren möglichen cut-Herstellung, nämlich den Realisierungsversuch, ob auch die Angabe über die Vergangenheit dadurch mitbestimmt wird. 
Aus klassisch experimentalpsychologischer Sicht sollte die als stabil angenommene Ausprägung in den letzten Jahren nicht an sich von der Reihenfolge der Beantwortung abhängen. Sollten sich bei einer Aussage über die Vergangenheit unterschiedliche Antworten in Abhängigkeit von der Reihenfolge ergeben, würde dies als Zeichen für mangelnde Reliabilität und Validität der Messung gewertet. Aus ARqE-Perspektive ist hingegen eine Messung, die das Ergebnis mitbestimmt, nie im klassischen Sinne (in)valide, sondern offenbart damit das Mit-Konfigurieren.

Es wird also außerdem nach der geschlechtlichen Selbstkategorisierung in den letzten Jahren gefragt. Da nicht von Stabilität ausgegangen wird und dies auch den Studienpartner_inne_n nicht suggeriert werden soll, wird das Wort „meistens" verwendet, um nahezulegen, dass diese Empfindung plausiblerweise variiert haben kann und nur eine Art Durschnittsangabe erwartet wird. Hier soll nicht verglichen werden, ob die Frage nach der Vergangenheit in nonbinärer Abfrage andere Antworten realisiert als in binärer Abfrage, sondern, ob die Reihenfolge das Ergebnis mitbestimmt. Die Vergangenheitsfrage wird daher nur in der Frageart realisiert, die variantenreichere Antworten realisieren sollte, nämlich in nonbinärer Frageart.

Realisiert wird die binäre Abfrage durch die klassische Frage der Form „Ihr Geschlecht:" mit der Präsentation von zwei Ankreuzalternativen, von denen eine mit „weiblich“ und die andere mit „männlich“ bezeichnet ist. Die nonbinäre Frageform präsentiert dagegen als Antwortformat eine Linie deren Endpunkte mit „weiblich“ und „männlich“ versehen sind. Auf dieser Linie soll ein Kreuz gesetzt werden. Es gibt neben der Linie außerdem die Option, „weder noch“ anzukreuzen.

Damit die Frage nach der Vergangenheit nicht lautet "Ihr Geschlecht in den letzten Jahren meistens?“, formulierte ich wie folgt: „Wie fühlten Sie sich in den letzten Jahren meistens?“. Analog dazu lautet die nonbinäre Frage nach der aktuellen Selbstkategorisierung, wie sich die Studienpartner_innen fühlen. 
In der nonbinären Variante werden also die beiden Fragen gestellt: „Wie fühlen Sie sich jetzt gerade? Setzen Sie ein $\times$ :" und „Wie fühlten Sie sich in den letzten Jahren meistens? Setzen Sie ein $\times:$ :.

Zusätzlich zu diesen Konfigurationen soll erprobt werden, ob sich bewertende Angaben über diese Fragen realisieren lassen. Döring (2013) beschreibt in ihrer Nennung der instrumentellen Reaktivität, dass diese möglicherweise zu einer erwünschten Wirkung führen könnte: „Da eine Befragung (...) auch eine Intervention (...) darstellt (...), mag angesichts der kulturellen Dominanz des Modells der Zweigeschlechtlichkeit eine gewisse Irritation [gegenüber mehr als zwei Antwortvorgaben] bei den Befragten durchaus wünschenswert sein." (Döring, 2013, S. 103) Damit deutet Döring eine Strategie an, um heteronormative Geschlechterbinarität lokal und temporär ein wenig aufzubrechen: Wenn wir die Geschlechtsabfrage mit mehr oder anderen als der klassischen Antwortmöglichkeiten von „weiblich“ und „männlich“ ausstatten, könnte dies die Zweigeschlechtlichkeit entselbstverständlichen. Allerdings befürchtet Döring als gleichzeitige Wirkung unerwünschte negative Einstellungen bei den Studienpartner_inne_n gegenüber der Frageart oder der Studie:

Es ist jedoch empirisch abzuklären, wie eine wenig sensibilisierte Zielgruppe Geschlechts-Items mit mehr als zwei Ausprägungen aufnimmt. Der Wirkung eines vielleicht wünschenswerten Denkanstoßes steht die mögliche unerwünschte Nebenwirkung negativer Einstellungen gegenüber dem Forschungsteam bzw. -projekt gegenüber, etwa der Eindruck, es würden unsinnige Fragen gestellt und die Studie sei dubios. (Döring, 2013, S. 103)

Neben einer Irritation lautet ihre zweite Wirkungsthese also, dass die nonbinäre Geschlechtsabfrage dazu führen könnte, dass Studienpartner_innen negative Einstellungen gegenüber der Frageform, der Studie insgesamt oder gegenüber den Forschenden entwickeln.

An dieser Stelle könnte die Beschreibung einer Studie der klassischen Experimentalpsychologie nahtlos dazu übergehen, dass diese These hier getestet werden soll und daher die entsprechenden Einstellungen nach der jeweiligen 
Geschlechtsabfrage erhoben werden. ARqE strebt dagegen an, die eigenen in der Forschung gesetzten agential cuts zu thematisieren. Daher ist eine Diskussion des Konstruktes vorgeschaltet:

Wenn wir versuchen wollen, diese Realisierung zu erproben, stellt sich aus ARqE-Perspektive die Frage, ob wir solche Einstellungen, wenn sie messbar würden, nicht erst durch unsere Nachfrage generieren. Selbst innerhalb der Psychologie existiert eine gewisse Debatte darüber, ob Einstellungen stabile kognitive Zustände (mental states) oder im Moment der Abfrage gebildete evaluative Urteile sind (Schwarz \& Bohner, 2001). Ferguson und Zayas (2009) sammeln Beispiele, wie häufig eine Evaluation die Verarbeitung eines Stimulus' begleitet - was sie automatic evaluation nennen -, ohne dass eine konkrete Abfrage die Einstellung erst herstellen würde. Schwarz und Kollegen (z. B. Schwarz und Bohner, 2001; Schwarz und Strack, 1991) vertreten dagegen folgenden Standpunkt: "[A]Il we assess in attitude measurement are evaluative judgments that respondents construct at the time they are asked, based on whatever information happens to be accessible." (Schwarz und Bohner, 2001, S. 437) Aus ARqEPerspektive stimme ich mit dem ersten Teil des Zitats überein, dass die evaluativen Urteile von Studienpartner_inne_n erst zum Zeitpunkt der Abfrage erstellt werden - allerdings im Sinne von Barad (2007) als basierend auf den speziellen Konfigurationen, mit denen ein Apparat realisiert ist, bzw. im Sinne von Butler als based on the field of recognizability (vgl. Butler, 2015, S. 38). Zu den Ergebnis-verursachenden Konfigurationen gehört aus ARqE-Perspektive nicht nur, welche Informationen zugänglich sind, sondern auch alle früheren oder indirekteren Einflüsse, die eine Information so gestalten, wie sie im Moment erscheint. ${ }^{75}$

Aus Perspektive der ARqE muss ich also unsicher sein, ob nicht erst eine Nachfrage im Fragebogen eine Bewertung (z. B. „unsinnig“ oder „dubios“)

75 Unter Umständen würden Schwarz und Kollegen dies auch nicht verneinen. Der ARqE ist es jedoch besonders wichtig, die Abhängigkeit von Ergebnissen (wie einer konkreten Evaluation) von größeren Zusammenhängen zu betonen, und Formulierungen nicht so klingen zu lassen, als ob ein mentaler Zustand nur Ergebnis der Rechenleistungen eines einzelnen kognitiven Apparates wäre. 
realisiert. Es könnte sein, dass allein die Überraschung über ungewohnte Fragen eine Bewertung realisiert, die wir als Forschende dann tatsächlich nur noch messen - mit zu vernachlässigender Mitgestaltung. Ausgehen kann ich davon aber nicht. Meines Erachtens wäre es interessant und wichtig, konkrete Einstellungsforschung aus ARqE-Perspektive durchzuführen, um ein ARqE-Verständnis über die agential cuts entwicklen zu können, die Einstellungen realisieren. Im Moment kann ich nur versuchen, vorzuführen, dass bei Personen auf Abfrage eine Bewertung zu realisieren ist.

Dieses Ziel ist ein anderes als die Hypothese von Döring. Letztere besteht darin, empirisch zu zeigen, dass A (ungewöhnliche Fragen) zu B (diese Fragen/ Studie dubios zu finden) führt. Sollten sich bestimmte Einstellungen realisieren lassen, lautet die Einsicht aus Perspektive der ARqE dagegen: Wenn wir B (Fragen/ die Studie als beispielsweise dubios zu benennen) die Möglichkeit zur Realisierung geben (wir fragen danach, wie die Fragen empfunden werden, und tun dies auf bestimmte Art und Weise), kann sich B durch A (es sind situativ ungewöhnliche Fragen) und den eröffneten Möglichkeitsraum (dass und wie wir danach fragen; dass es kulturell verbreitete Ideen $\mathrm{zu}$ den verwendeten Konzepten gibt; was die individuellen Konzepte sind; etc.) realisieren.

Problematisch ist an diesem Vorgehen, dass möglicherweise ohne unsere Abfrage situativ durch keine anderen Intra-aktionen eine bestimmte Bewertung hergestellt würde. In diesem Fall würde ich als Forschende die Äußerung einer negativen Einstellung gegenüber einer nonbinären geschlechtlichen Selbstkategorisierung erst hervorbringen. Womöglich würden die Studienpartner_innen ohne meine Abfrage meine Studie am Ende auch ohne negative Bewertung der nonbinären Geschlechtsabfrage verlassen.

Solche Fragen würden in der klassischen Experimentalpsychologie nicht gestellt. Vielmehr würde davon ausgegangen, dass die negative Bewertung unabhängig von der Frage danach evoziert würde.

Im Bewusstsein dieser möglichen Problematik, durch die Studie das Phänomen Einstellung gegenüber untypischer Geschlechtsabfrage erst herzu- 
stellen, soll hier vorgeführt werden, dass bestimmte Bewertungen der nonbinären Selbstkategorisierung realisiert werden können.

Ich benutze bewusst die Formulierung bestimmte Bewertungen und nicht negative Bewertungen, da ich die Klassifizierung negativ als eine weitere, erst situativ gebildete Kategorisierung erachte.

Selbstverständlich stellt jedes Wort eine situierte Kategorisierung dar, die man problematisieren kann - man kann beispielsweise auch fragen, ob die Stu-dienpartner_innen untereinander teilen, was sie unter Fragen empfinden verstehen. In dieser Studie setze ich agential cuts auch dadurch, dass ich annehme, dass bezüglich des Verständnisses, wie man Fragen empfindet, unter meinen Studienpartner_inne_n größerer und unproblematischerer Konsens besteht, als bei der Frage, welche Adjektive als negativ gewertet werden. Nachfolgende Forschung könnte meine gesetzen cuts wiederum problematisieren und meine Selbstverständlichkeiten in Frage stellen. Hier stelle ich zunächst die Praktik in Frage, daraus, dass bestimmte Adjektive von bestimmten Gruppen in bestimmten Fragebögen als negativ eingeschätzt werden, zu schließen, dass meine Studienpartner_innen diese Adjektive als negativ empfinden. Üblicherweise legen Psycholog_innen Studienpartner_innen Listen von Adjektiven zur Einschätzung vor, um festzulegen, welche sie in Folgestudien als kulturell negativ bewertet u. a. verwenden. Tatsächlich handelt es sich hier - weil es keine Operationalisierung von negativ oder dubios gibt (vgl. Kap. 4.2.3) - lediglich um die Feststellung eines Zusammenhanges zwischen zwei konkreten Antwortverhalten.

Ich wähle Adjektive, die situativ als positive oder negative Beschreibungen empfunden werden können. Dabei gehe ich davon aus, dass diese Einschätzung inter-individuell, situativ intra-individuell und auch in Abhängigekeit davon, worauf sich ein Adjektiv bezieht, variiert. Daher soll keine Aussage über Positivität oder Negativität der Bewertungen getroffen werden. Es werden lediglich sieben Eigenschafts-Dimensionen ausprobiert, auf denen die Studienpartner_innen je auf einer 7-stufigen Likert-Skala angeben sollen, wie sie die jeweilige Geschlechtsabfrage empfinden. Dabei habe ich versucht, jeweils Gegensatzpaare zu finden - wobei nicht vorauszusetzen ist, dass die Studienpartner_innen dies ebenso empfinden. Die 
Paare lauten: unseriös-seriös, interessiert-oberflächlich, befremdlichangenehm, menschenfreundlich-menschenverachtend, engstirnigweltoffen, realitätsnah-realitätsfern, sachlich-unsachlich. Außerdem wird die offene Frage gestellt: „Die Fragen wirken außerdem:“, worauf die Studienpartner_innen mit einem Freitext antworten können.

Die Wahl von Likert-Skalen mit bestimmten Bezeichnungen wie ,unseriös“ und „oberflächlich“ stellt hier keine Operationalisierung in dem Sinne dar, dass diese Adjektive Indikatoren für den Sachverhalt als-dubios-empfinden wären. Vielmehr werden Adjektive ausprobiert und erprobt, ob die Zustimmung zu jenen systematisch von der Frageform nach Geschlecht und/ oder der Reihenfolge mitbeeinflusst wird.

Die Richtung einer solchen Herstellungs-Hypothese würde aus der genauen Betrachtung der agential schneidenden Konfigurationen entstehen. Aus Kenntnis über lokal und temporär wirkende Heteronormativität, die eine geschlechtliche Kategorisierung hin zur Binarität beschränkt, könnten Richtungen für jede Herstellungs-Hypothese jeder Adjektiv-Einschätzungs-Dimension gebildet werden. Ohne hinreichendes Vorwissen über situative Intra-aktionen haben wir jedoch kaum Anhaltspunkte für Generierung von Hypothesenrichtungen. Hier soll mangels Vorwissen über dieserorts potentiell wirkende Konfigurationen (neben Heteronormativität) aus $A R q E$-Perspektive zunächst ungerichtet getestet werden, auch wenn ich spekulierende Annahmen äußern kann.

Unterschiedshypothesen teste ich - mangels Vorwissen aus ARqEPerspektive - zunächst ungerichtet. Zusätzlich kann ich jedoch in einem zweiten Schritt Vermutungen über wirkende Intra-aktionen auf Basis einer heteronormativen Prägung unserer aktuellen Kultur verwenden und sehen, ob sich die u. a. dadurch vorhergesagten Richtungen der Unterschiede realisieren lassen.

Für die sieben Dimensionen werden also zusätzlich die folgenden spezifizierenden Thesen aufgestellt: 
- Weil unsere aktuelle Kultur heteronormativ geprägt ist, wird die binäre Frageform als seriöser als die nonbinäre markiert.

- Durch die Bereitstellung von mehr Antwortalternativen evoziert die Dimension interessiert-oberflächlich die Beurteilung der nonbinären Frage als interessierter als die binäre Frageform.

- $\quad$ Aufgrund der Bekanntheit und Gewöhnung wird die binäre Frageform eher als angenehm als die nonbinäre markiert.

- Aufgrund der Bereitstellung von mehr Antwortalternativen, die die klassisch binären nicht ausschließen, wird die nonbinäre Frageform als menschenfreundlicher als die binäre markiert.

- $\quad$ Aus demselben Grund wird die binäre Frageform als weniger weltoffen, als die nonbinäre markiert.

- Aufgrund der heteronormativen Prägung unserer Kultur wird Geschlechterbinarität als gegeben erachtet und die binäre Frageform als realitätsnäher als die nonbinäre markiert.

- Analog zur Seriosität wird die binäre Frageform als sachlicher als die nonbinäre markiert.

Selbst wenn sich das Ergebnis realisieren lässt, dass die binäre Frageform durch diese Konfigurationen des Fragebogens als realitätsnäher als die nonbinäre eingestuft würde, muss die entscheidende mitbestimmende Intraaktion nicht die vermutete heteronormative Prägung unserer Kultur, aufgrund derer Geschlechterbinarität als gegeben erachtet wird sein. Vielmehr ist diese Vermutung eine, an der sich nachfolgende Studien orientieren könnten. Zunächst ist lediglich die Realisationsmöglichkeit auf dem Prüfstand.

Um den Fragebogen ähnlich zu einem in den Sozialwissenschaften üblichen zu machen, wird darin nicht nur nach Geschlecht von Studienpartner_innen gefragt, sondern auch nach anderen als "major demographic characteristics" (APA, 2010, S. 29) bezeichneten Personvariablen: "age; (...) ethnic and/or racial group; level of education; socioeconomic, generational 
or immigrant status, (...)" (APA, 2010, S. 29). Aus queertheoretischer Sicht ist jedoch die unproblematisierte Reproduktion solcher Konstrukte als aussagefähige einzelne Variablen kritisch zu sehen. Aus einer queeren Perspektive ist es problematisch, wenn ein wissenschaftlicher Fragebogen den Studienpartner_inne_n vermittelt, dass durch die Frage nach der Muttersprache ein wissenschaftlich erklärungsrelevantes Konzept erfasst würde. Diese Frage wird in psychologischen Studien nicht selten dazu verwendet, die Daten jener, die als Muttersprache nicht die des Fragebogens angeben, aus der Analyse auszuschließen. Dieses Vorgehen wird damit begründet, dass jene die Fragen und deren Konnotationen möglicherweise nicht korrekt verstehen würden. Eine aus queertheoretischer Sicht akzeptable Begegnung dieser Forscher_innen-Befürchtung wäre, die Studienpartner_innen zu fragen, wie gut sie die Fragen der Studie verstehen. Dies lässt die Option offen, die Daten derer, die einen bestimmten Grad an Verständnisschwierigkeiten angeben, nicht in die Datenanalyse einzubeziehen; gleichzeitig schreibt das Vorgehen den Studienpartner_inne_n Kompetenz zu, diesen Punkt selbst beurteilen zu können, statt das Kriterium für den Verständnisgrad von extern an Drittvariablen festzumachen. Solche Überlegungen sind aus ARqE-Perpektive für jede der vermeintlichen major demographic characteristics anzustellen.

In einer Studie, die Aussagen über lokal relevante, soziale Kategorien machen möchte - wie zum Beispiel als Frau kategorisierte Personen -, könnten diese weiteren sozialen Kategorien wichtig werden, um die eigentlich interessierende Kategorie intersektional zu betrachten. Im vorliegenden Fall werden nur mehrere Antwortverhalten innerhalb der gleichen Stichprobe in Zusammenhang gebracht. Die Intersektionalität von Gruppenzugehörigkeiten wäre erst dann zu bedenken, wenn von der befragten Stichprobe auf eine angenommene Population verallgemeinert werden sollte. Diese Generalisierung wird hier nicht unternommen (siehe untenstehender Abschnitt der Interpretation ab Seite 226). Dass es sich aber insgesamt nur um lokal und temporär gültige Konzepte handelt versteht sich von selbst. 
Um die klassische Frageform nach Alter, Geschlecht, Staatsangehörigkeit, Mutterprache den Studienpartner_inne_n nicht alleinstehend und unproblematisiert zu präsentieren, nimmt der Fragebogen auch alternative, queertheoretisch kompatiblere Frageformen auf. Wieder sollen aus ethischen Gründen alle Studienpartner_innen alle Fragen vorgelegt bekommen, damit nicht eine Stichprobe nur die queertheoretisch problematischen erhält.

Auch hier könnten solche Erklärungen bei Voraussetzung der ARqEPerspektive als bekannt wegfallen. Analog wird in klassischen Studien auch nicht jedes Mal die klassische Forschungslogik erklärt. Hier dienen diese Erklärungen der momentanen Verständlichkeit. Im folgenden Abschnitt zeigt sich, dass aus ARqE-Perspektive als relevant erachtet wird, mit welchem Material die Studienpartner_innen konfrontiert werden. Auch hier ist die Gestaltung der Frage sehr wahrscheinlich eine Ergebnis-mitbestimmende Konfiguration.

Bezüglich des Alters ist aus queertheoretischer Sicht die Position naheliegender, dass Alter keine entscheidende alleinstehende Personenvariable mit bestimmten Konsequenzen ist, sondern dass kultureller Umgang sogenannte Altersphänomene moderiert. Wenn eine ältere Person die Handhabung moderner Technik schwierig findet, liegt dies demnach nicht direkt am Alter, sondern daran, wie Gesellschaft Strukturen gestaltet, und daran, dass moderne Technik so gemacht ist, dass ältere Menschen sie tendenziell schlechter handhaben können als jüngere. Aus queertheoretischer Perspektive ist viel weniger relevant, wie alt eine Person kalendarisch ist, als dass sie durch kulturelle Handlungen jung, normal oder alt gemacht wird. Entsprechend würde ein queerinformierter Fragebogen nicht nach dem Alter fragen, sondern nach den Faktoren, die kulturell Alter zu einer intelligiblen Variable machen. Ein solche Frage könnte z. B. lauten: „Wodurch werden Sie kulturell so jung/alt gemacht, wie Sie gemacht werden?" Allerdings ist anzunehmen, dass Personen unserer heteronormativ geprägten Kultur ohne queertheoretische Bildung diese Frage nicht ohne Weiteres im queertheoretisch intendierten Sinne verstehen würden. Als Alternative werden hier folgende Fragen erprobt: „Fällt Ihnen Ihr Alter im Alltag auf?“ 
und anschließend „Warum, denken Sie, ist das so?" Als wünschenswert erachte ich, wenn Menschen durch diese Fragen dazu gebracht werden, darüber nachzudenken, warum ihnen ihr Alter im Alltag auffällt oder nicht, und wenn sie dadurch möglicherweise auf die Idee kommen, dass es ihnen auffällt, weil sie gesellschaftlich unnormalisiert werden, bzw. dass es ihnen nicht auffällt, weil sie normalisiert werden. Wiederum ist es allerdings wie bei der versuchten Realisierung von Geschlechterdiversität - nicht allzu wahrscheinlich, dass zwei kurze Fragen den übrigen (und üblicheren) Konfigurationen entgegenwirken können. Viel wahrscheinlicher ist, dass die meisten Studienpartner_innen - wie gewohnt - das Auffallen oder Nicht-Auffallen auf sich selbst zurückführen und nicht auf den Kontext. Sie werden vermutlich antworten, dass es ihnen beispielsweise nicht auffällt, weil sie noch nicht zu alt sind, bzw. dass es ihnen auffällt, weil sie alt sind. Möglicherweise werden nur jene, die sich normal fühlen, aber in Deutschland nicht den altersmäßig Normalisierten entsprechen, eine Ausgrenzung angeben können. So könnten sich beispielsweise 17- und 18-Jährige des großen Qualitätssprungs der Volljährigkeit bewusst sein, obwohl die gefühlten Veränderungen als klein wahrgenommen werden. Sie könnten vermutlich eher benennen, dass nicht das Alter als Personenvariable etwas bewirkt, sondern nur $^{76}$ in einer Interaktion (bzw. Intra-aktion) mit gesellschaftlichen Zuschreibungen.

Durch die Aufnahme von alternativen Frageformen zu den Konstrukten Alter, Sprachkenntnisse, Gruppenzugehörigkeit, sozioökonomischer Status und Religionszugehörigkeit, soll den Studienpartner_inne_n im hier verwendeten Fragebogen vorgeführt werden, dass sozialwissenschaftliche Fragen auch anders als in klassischer Weise gestellt werden können - auch wenn nicht zu erwarten ist, dass dies sogleich entselbstverständlichende Auswirkungen hätte. Aus ökonomischen Gründen werden in dieser Studie keine weiteren Phänomenrealisierung (wie Entselbstverständlichung) ver-

\footnotetext{
76 In einigen anderen Fällen ist Alter nur eine Trägervariable (vgl. Trautner, 1992), in denen der Effekt gar nicht vom eigentlichen Alter abhängt, sondern z. B. von der Sozialisation einer bestimmten Alterskohorte (Beispiel: Menschen bestimmten Alters sind nicht mit Facebook aufgewachsen).
} 
sucht und die Aufnahme der alternativen Frageformen dient eher der Anregung, sich in zukünftigen Studien kritischer mit major demographic characteristics zu beschäftigen. Ebenso aus ökonomischen Überlegungen heraus platziere ich nicht jede Frage im Kontext jeder anderen. Stattdessen beinhaltet der Fragebogen einen Block mit klassisch formulierten Fragen inklusive der binären Geschlechtsabfrage und einen Block mit alternativ formulierten Fragen inklusive der nonbinären Geschlechtsabfrage.

Döring (2013) äußert die Befürchtung, dass durch eine ungewöhnliche Geschlechtsabfrage die ganze Studie für dubios befunden werden könnte. Ich werfe die Frage auf, welche Einschätzungen dieser einzelnen Frage - im Vergleich zu den anderen des Fragebogens - realisiert werden können. Ist es möglich, die Antwort zu realisieren, dass die nonbinäre Geschlechtsabfrage als besonders eigenartig eingeschätzt wird? Die Gewöhnlichkeit der binären Geschlechtsabfrage in unserer aktuellen Kultur könnte nachfolgende cuts mitbestimmen. Auf den oben beschriebenen Nachfragen per Likert-Skalen könnte die binäre Geschlechtsfrage als noch seriöser eingestuft werden als der Block, der Fragen nach Einkommen und Religionszugehörigkeit beinhaltet, während eine ungewohnte Geschlechtsabfrage in demselben Vergleich als noch unseriöser eingestuft werden könnte. Ich sage eine Interaktion dieser Richtung auf der Dimension Seriösität voraus. Auch die anderen Adjektiv-Skalen sollen zur Anwendung kommen, wobei hier ungerichtet getestet wird. Es soll also eine Hälfte der Studienpartner_innen die Adjektiv-Skalen in Bezug auf den ganzen vorangehenden Fragenblock beantworten und die andere Hälfte nur in Bezug auf die Geschlechtsabfrage. Da mir diese unterschiedliche Konfrontation der Studienpartner_innen queertheoretisch unproblematisch erscheint, kann diese Bedingung interindividuell variieren um nicht - wie bei einer intraindividuellen Variation - die Studienpartner_innen auf diesen Unterschied aufmerksam zu machen und dadurch womöglich einen weiteren agential cut zu evozieren.

Das Gesamtdesign sieht aus wie folgt: Die Frage nach der geschlechtlichen Selbstkategorisierung wird jeder_jedem Studienpartner_in einmal klassisch binär und zweimal nonbinär formuliert gestellt, wobei sich im nonbinären 
Fall die erste dieser Fragen auf die jetzige und die zweite auf die häufigste Selbstkategorisierung der letzten Jahre bezieht. Beide Geschlechtsabfrageformen finden sich im Kontext der Abfrage weiterer von der APA als major demographic characteristics verstandenen Konstrukte wie Alter, sozioökonomischer Status und Religion. Damit ergeben sich ein Block klassisch formulierter Fragen und ein Block alternativ formulierter Fragen. Nach diesen Blöcken beantworten die Studienpartner_innen jeweils die Likert-Skalen zur Bewertung der vorherigen Frage(n), wobei die Hälfte der Studienpartner_innen dies für den gesamten vorherigen Fragenblock beantworten soll (im Folgenden „Bezug alle“), und die andere Hälfte nur für die Geschlechtsabfrage(n) (im Folgenden „Bezug G“). Die Reihenfolge der (klassischen und alternativen) Blöcke ist für eine Hälfte der Studienpartner_innen erst klassisch, dann alternativ (im Folgenden „Rf kl_alt“) und für die andere Hälfte erst alternativ, dann klassisch (im Folgenden „Rf alt_kl“).

Empirisch erwarte ich vorführen zu können, dass in den Antworten der Studienpartner_innen auf die nonbinäre Geschlechtsabfrage mehr Geschlechtervarianz zu finden ist als in den Antworten auf die binäre Frageform. Darüberhinaus könnte dieses Ergebnis von der Reihenfolge abhängen. Außerdem könnte die nonbinäre Frageform bezüglich bestimmter Adjektive anders eingestuft werden als die binäre Frageform. Im zweiten Schritt sollen bezüglich der Adjektiv-Zustimmung auch gerichtete Hypothesen betrachtet werden. Des Weiteren sollte die nonbinäre Geschlechtsabfrage als unseriöser als der gesamte alternative Fragenblock bewertet werden, aber die klassische Geschlechtsabfrage als seriöser als der gesamte klassische Fragenblock.

Um eine Stichprobe zu erhalten, die queertheoretisch nicht besonders vorgebildet ist - bzw. wie Döring es nennt: „eine wenig sensibilisierte Zielgruppe“ (Döring, 2013, S. 103) - wurde der Fragebogen im April 2014 an Fußgänger_innen eines belebten öffentlichen Ortes (Kölner Rheinpromenade $^{77}$ ) ausgeteilt. Kinder wurden als Studienpartner_innen ausgeschlos-

77 Obwohl in Köln eine umfangreiche Lesben- und Schwulenszene existiert (vgl. Wikipedia, 2016), ist vom Publikum an der Rheinpromenade keine besondere queertheoretische Prägung anzuneh- 
sen, damit sie das Konzept der geschlechtlichen Selbstkategorisierung nicht wiederholen. Alle Studienpartner_innen wurden informiert, dass es sich um einen Fragebogen für eine Doktorarbeit handelt. Als Dankeschön für die Beteiligung erhielten die Studienpartner_innen einen Schokoladenriegel. Da die Beschreibung der Studie - dass es darum geht zu erfahren, wie Fragebögen auf Menschen wirken - der Wahrheit entsprach, gab es keine gesonderte Aufklärung nach Beendigung der Studie. Doch jede_r Studienpartner_in hatte die Möglichkeit, ihre_seine Kontaktdaten auf einem separaten Formular zu hinterlassen, um die Ergebnisse der Studie zu erfahren.

\subsection{Ergebnisse}

Hier wird für die einzelnen Ergebnismuster der Dreischritt Ergebnisse Interpretation - Diskussion vorgeschlagen, sodass zunächst ein realisiertes Ergebnis sehr technisch besprochen werden kann, danach eine Interpretation vorgeschlagen wird und in der Diskussion weitere Implikationen der Interpretationen diskutiert werden.

Es wurden 100 Fragebögen an Personen ausgegeben, die sich zunächst bereit erklärten, den Fragebogen auszufüllen. Die einzig intendierte Fremdkategorisierung der Studienpartner_innen durch die Studienleitung bezog sich darauf, keine Kinder anzusprechen. Eine Person brach das Ausfüllen mit der Begründung ab, dass die Fragen zu persönlich seien. Nicht in die Analyse aufgenommen wurden die Daten von Studienpartner_innen, die die Frage, wie gut sie die Fragen des Bogens verstehen, entweder nicht beantwortet (3 Personen) oder als erheblich angenommene Verständnisprobleme angegeben hatten (auf der Skala entsprach 1 einem problemlosem Verständnis, 7 Verständnisproblemen; als erhebliche Ver- 
ständnisprobleme wurde ein Ankreuzen in der Mitte der Skala oder darüber hinaus - d. h. $>3$ - festgelegt).

Statt den Ausschluss aus der Analyse über die Muttersprache zu regulieren, wird hier die Frage verwendet, ob die Studienpartner_innen die Fragen des FB problemlos verstehen. Dadurch wird versucht, das eigentlich interessierende Kriterium - die Konnotationen des Fragebogens im Sinne der_des Forschenden zu verstehen - abzufragen und nicht von der Drittvariable Muttersprache abzuleiten, dass alle Deutsch-Muttersprachigen die Konnotationen problemlos verstehen und alle Nicht-Deutsch-Muttersprachigen diese nicht verstehen würden. ${ }^{78}$

In die Analyse gingen die Daten von 94 Studienpartner_innen ein, die sich

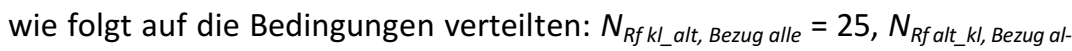
$l e=23, N_{R f k l \_a l t, \text { Bezug G }}=22, N_{R f a l t \_k l, \text { Bezug } G}=24$.

\section{Form der Geschlechtsabfrage}

Die klassische binäre Abfrage produzierte Antworten (mit), die zu 100\% im binären Schema verblieben, das heißt alle Antworten lauten entweder „weiblich“ oder „männlich“.

Eine solche Formulierung sollte charakteristisch für die ARqE werden. Absichtlich wird nicht konstatiert, dass „keine der Studienpartner_innen das binäre Antwortschema verlassen hat", um die Ursache des Ergebnisses nicht zu sehr in den Studienpartner_innen zu lokalisieren. Aus ARqE-Perspektive führt eine Vielzahl von agentials cuts die zu einer Realisierung. Somit ist es passender, die Ursache in der Gesamtkonfiguration zu lokalisieren. Genau genommen sollte sogar der Zusatz ,in diesem Fragebogen, der von jener Stichprobe zu jener Zeit an jenem Ort beantwortet wurde" beigefügt werden. Da die Zeitlichkeit und Lokalität in der ARqE jedoch stets betont wird, wird hier darauf verzichtet. Es wird außerdem die Vergangenheitsform verwendet, um nicht mit der Gegen-

788 Personen haben als alleinige Muttersprache eine andere als ,Deutsch` angegeben und trotzdem auf der Verständnisskala eine 1 oder 2 (problemloses Verständnis) angekreuzt. Die Daten jener würden in einer klassischen Studie wahrscheinlich aus der Analyse entnommen. 4 weitere Personen hatten neben Deutsch eine weitere als Muttersprache angegeben - alle davon gaben eine 1 auf der Verständnisskala an. Andersherum gab eine_r von den beiden, die Unverständnis markierten, Deutsch als Muttersprache an. 
wartsform zu implizieren, dass das Beschriebene immer so sei. Es ist jedoch wünschenswert, dass zukünftig nach weiteren bzw. treffenderen situierenden Formulierungen gesucht wird.

Es wäre denkbar gewesen, dass die alternative Abfrageform - zumindest in der Reihenfolgenbedingung, in der die nonbinäre Abfrage vor der binären erfolgt - fördert, dass Studienpartner_innen in der binären Abfrageform die vorgegebenen zwei Kästchen missachten und z. B. ein drittes hinzumalen. Dies geschah in keinem Fall. Auch wurde die Beantwortung dieser binären Frage nie ausgelassen.

Die Antworten auf die alternative Geschlechtsabfrage habe ich folgendermaßen quantifiziert. Das Kontinuum wurde in drei gleich große Bereiche eingeteilt und zunächst vermerkt, ob ein_e Studienpartner_in sein_ihr Kreuz auf den als weiblich bezeichneten Punkt (1) setzt, in das erste Drittel des Kontinuums nahe „weiblich“ (2) , in das mittlere Drittel (3), in das dritte Drittel nahe „männlich“ (4), oder auf den als männlich bezeichneten Punkt (5), oder ob die_der Studienpartner_in die Alternative „weder noch“ (6) ankeuzte. Alle Antworten, in denen nicht direkt der als weiblich oder der als männlich bezeichnete Punkt markiert wurde (2, 3, 4 und 6), wurden als geschlechterdiverse Antwort gezählt. Wurde exakt der als weiblich bezeichnete (1) oder der als männlich bezeichnete Endpunkt (5) angekreuzt, wurde diese Antwort als ,verbleibt in Binarität“ gewertet.

Zugegebenermaßen impliziert der Ausdruck „verbleiben“ eine Bewegungsrichtung (und wird ggf. als wertend empfunden). Ich wähle den Ausdruck dennoch, da Geschlechterbinarität in einer heteronormativ geprägten Gesellschaft der gängige Zustand ist und eine nonbinäre Antwort das darin Untypische darstellt. Jedoch wird im Text auch darauf geachtet, dass von Antworten und nicht von Personen die Rede ist, um nicht die Personen zu essentialisieren.

Auf der alternativen Geschlechtsabfragedimension bezüglich "jetzt“ keuzten insgesamt 45 Personen (47,9\%) eine von mir als geschlechterdivers kategorisierte Antwort an. Eine Person gab keine Antwort. Die Antworten von 48 Personen (51,1\%) verblieben in der Binarität. Bei der 
Selbstkategorisierung bezüglich „der letzten Jahre“ wählten insgesamt 46 Personen (48,9 \%) eine geschlechterdiverse Antwort und die Antworten von 48 Personen $(51,1 \%)$ verblieben in der Binarität. Die Anworten von 45 Personen (47,9\%) blieben bei beiden alternativen Geschlechtsabfragen in der Binarität und die Antworten von 42 Personen (44,7 \%) wurden beide Male als geschlechterdivers kategorisiert. Bei den Antworten von 6 Personen $(6,4 \%)$ war nur eine der zwei Antworten binär und die andere geschlechterdivers. Fast die Hälfte der befragten Studienpartner_innen wählten also auf diesen alternativen Geschlechtsabfragen Antworten, die als geschlechterdivers kategorisiert wurden. Von den 45 Personen, die bezogen auf "jetzt" eine geschlechterdiverse Antwort gaben, kreuzten 17 Personen im ersten Drittel nahe weiblich an, $5 \mathrm{im}$ mittleren Drittel des Kontinuums, 14 im dritten Drittel nahe männlich und 8 „weder noch“. Eine Person kreuzte die Mitte des Kontinuums und das „weder noch" an. Von den 46 Personen, die bezogen auf „in den letzten Jahren meistens“ eine geschlechterdiverse Antwort gaben, kreuzten 20 Personen im ersten Drittel nahe weiblich an, $7 \mathrm{im}$ mittleren Drittel des Kontinuums, $13 \mathrm{im}$ dritten Drittel nahe männlich und 5 „weder noch“. Eine Person schrieb „sowohl als auch" auf das Kontinuum und kreuzte zusätzlich „weder noch“ an.

Die Nennung von quantitativen Ergebnissen kann nahezu wie in klassisch experimentalpsychologischen Studien geschehen, jedoch ist aus ARqEPerspektive darauf zu achten, nicht schon Ergebnisursachen in den Studienpartner_innen zu lokalisieren. So darf nicht von „geschlechterdiversen Personen“ gesprochen werden, sondern von „den Personen, die bezogen auf XYZ eine geschlechterdiverse Antwort gaben“.

\section{Form der Geschlechtsabfrage und Reihenfolge}

Zunächst kann ich auch aus $A R q E$-Perspektive die Realisierung von bestimmten Antworten und Antwortmustern benennen. Im Abschnitt 4.2.3 habe ich andiskutiert, dass bestimmte Tests zur Prüfung einer Unterschiedshypothese auch aus Perspektive der ARqE angewendet werden können. Der Unterschied besteht darin, dass nicht von der Existenz eines wahren Wertes ausgegangen wird, und dass die Streuung, die bei Messwiederholungen in der Regel entsteht, 
nicht als Indikatorin zufälliger Messfehler verstanden wird, sondern als Ausdruck der zugehörigen Varianzmöglichkeiten - oben als Realisierungsraum bezeichnet. Die Anwendungslogik für einen Signifikanztest - entscheiden zu wollen, ob ein Wert mit einer bestimmten Wahrscheinlichkeit zu einer bestimmten Verteilung gehört - bleibt bestehen. Wenn wir also davon ausgehen, dass die - durch die spezielle Studienkonfiguration an dem Ort und in der Zeit mit allen Zusammenhängen, in denen sie durchgeführt wurde - platzierten agential cuts aus einem größeren Möglichkeitsraum Realisierungen herausschneiden bzw. Ergebnisse materialisieren, dann prüft ein solcher Test, ob ein gesetzter cut - im Sinne von lokal kausal - wahrscheinlich wirkungsvoll war oder nicht. In unserem konkreten Beispiel gehe ich davon aus, dass die durch den Fragebogen gesetzten (zusammen mit den gesellschaftlich und situativ bestehenden) cuts wirken und auf dem nonbinären Antwortformat mehr nonbinäre Antworten produzieren - nämlich 45 nonbinäre zu 48 binären Antworten - als auf dem binären Antwortformat - nämlich 0 nonbinäre zu 94 binären Antworten. Ich kann nun testen, ob auch der agential cut der Reihenfolge für die nonbinäre Antwortenproduktion wirkungsvoll ist.

Um festzustellen, ob die Anzahl der geschlechterdiversen Antworten mit der Reihenfolge zusammenhängt, wurde je ein Chi-QuadratUnabhängigkeitstest für die beiden alternativen Geschlechtsabfragen gerechnet.

Der Anteil der Personen, die für ",jetzt" eine geschlechterdiverse Antwort gaben, unterschied sich in Abhängigkeit von der Reihenfolge, $\chi^{2}(1, N=93)$ $=5.68, p=.017$. Ebenso unterschied sich der Anteil der Personen, die für "die letzten Jahre" eine geschlechterdiverse Antwort gaben, in Abhängigkeit von der Reihenfolge, $\chi^{2}(1, N=93)=4.26, p=.039$. Bei der Frage bezüglich "jetzt" waren in der Reihenfolge "klassisch-alternativ“ deutlich mehr Antworten $\left(n_{1}=28\right)$ geschlechterdivers, als dass entweder weiblich oder männlich gewählt wurde $\left(n_{2}=18\right)$, während in der Reihenfolge "alternativ-klassisch" deutlich weniger Antworten $\left(n_{1}=17\right)$ geschlechterdivers waren, als dass entweder weiblich oder männlich gewählt wurde $\left(n_{2}=30\right)$. Auch bei der Frage bezüglich der „letzten Jahre“ waren in der Reihenfolge 
„klassisch-alternativ" deutlich mehr Antworten $\left(n_{1}=28\right)$ geschlechterdivers, als dass entweder weiblich oder männlich gewählt wurde $\left(n_{2}=19\right)$, während in der Reihenfolge "alternativ-klassisch“ deutlich weniger Antworten $\left(n_{1}=18\right)$ geschlechterdivers waren, als dass entweder weiblich oder männlich gewählt wurde $\left(n_{2}=29\right)$. Auch zusammengenommen hing also die Anzahl der Antworten, die mindestens einmal über die Binarität hinaus gingen, von der Reihenfolge $a b\left(\chi^{2}(1, N=94)=7.21, p=.007\right)$.

\section{Bewertung der Fragen}

Zunächst wurde geprüft, ob die Bewertung auf den Likert-Skalen je nach Bezug (nur auf die Geschlechterfrage/n vs. auf den ganzen Frageblock) variiert, möglicherweise in zusätzlicher Abhängigkeit von der Frageform bzw. der Reihenfolge.

Auch die Varianzanalyse halte ich trotz der prinzipiellen Annahme der ARqE, dass die Streuung von einzelnen Mess-Werten zum relatum-withinrelations dazugehört, für weiterhin geeignet. Mit ihrer Hilfe kann auch hier, trotz Annehmen eines Realisierungsraumes, der Einfluss spezifischer Konfigurationen (als unabhängige Variablen) geprüft werden. Nichtsdestotrotz ist für die Zukunft zweifellos eine genaue Diskussion der einzelnen Voraussetzungen und weiteren Annahmen solcher Tests aus Perspektive der ARqE vonnöten.

Die Varianzanalysen über den Faktor „Frageart“ (binär oder nonbinär) und den messwiederholten Faktor "Bezug“ inklusive des Faktors „Reihenfolge“ als Kovariate zeigten keinen Einfluss des Faktors Bezug auf die Bewertung, auch nicht in Form einer Interaktion zwischen den Faktoren Bezug und Frageform. Lediglich für die Skala „menschenfreundlich“ zeigte sich eine (zudem effektschwache) Tendenz, dass die Geschlechterfragen (klassich binär wie alternativ nonbinär formuliert) menschenfreundlicher als der jeweilige gesamte Fragenblock bewertet wurden. Das Signifikanzniveau von $5 \%$ wurde jedoch nicht unterschritten $\left(F(1,82)=3.218 ; p=.076\right.$; part.Eta ${ }^{2}$ $=.038)$. Gemäß dieses Ergebnisses des Signifikanztests muss also von Zufälligkeit ausgegangen werden, auch wenn das deskriptive Muster bei der Bewertungsdimension "seriös" dem in der zweiten Überlegung vorherge- 
sagten Muster entsprich: Die alternative Geschlechtsabfrage wurde als weniger seriös angegeben, als der gesamte alternative Fragenblock, während die klassische Geschlechtsabfrage als seriöser als der gesamte klassische Fragenblock angegeben wurde $\left(F(1,86)=1.916 ; p=.170 ;\right.$ part.Eta ${ }^{2}=$ .022). Ich bleibe also bei der Nullhypothese, dass der Bezug keinen Unterschied für die Beantwortung auf den Likert-Skalen macht - die Geschlechterfrage also nicht als wesentlich unseriöser als der ganze Fragenblock angegeben wurde und sich auch bezüglich der Reihenfolge als Kovariate hier keine unterschiedliche Markierung zeigte. In Folge dessen wurden die Daten nicht weiterhin nach dem Bezug der Bewertungsfragen differenziert.

Abgesehen von der Dimension „menschenfreundlichmenschenverachtend“, ergaben die $t$-Tests für abhängige Stichproben für alle anderen Bewertungsfragen, dass die alternative Frageform signifikant anders als die klassische beantwortet wurde ( $\left.p^{\prime} s<.05\right)$. Nach BonferroniKorrektur des Alpha-Fehler-Niveaus muss man jedoch einschränken, dass sich auf der Dimension „engstirnig-weltoffen“ nur eine Tendenz abzeichnete $(p=.033)$, ebenso auf der Dimension "realitätsnah-realitätsfern“ ( $p=$ .047); die anderen Dimensionen zeigten trotz Alphafehlerkorrektur signifikante Unterschiede (alle $p^{\prime} s<.007$ ). Die Unterschiede zeigten sich außerdem in den (im zweiten Schritt überlegten) spekulierten Richtungen:

- $\quad$ Die klassische Frageform wurde als seriöser $(M=5.4 ; S D=1.643)$ als die alternative $(M=4.1 ; S D=1.790)$ bewertet.

- Die klassische Frageform wurde eher als oberflächlich $(M=4.14 ; S D=$ 1.856), die alternative eher als interessiert $(M=3.23$; $S D=1.796)$ bewertet.

- $\quad$ Die klassische Frageform wurde eher als angenehm $(M=4.62 ; S D=$ $1.511)$, die alternative eher als befremdlich $(M=3.59 ; S D=1.643)$ bewertet.

- $\quad$ Die klassische Frageform wurde als weniger weltoffen $(M=4.33 ; S D=$ 1.641) als die alternative $(M=4.79 ; S D=1.496)$ bewertet. 
- $\quad$ Die klassische Frageform wurde als realitätsnäher $(M=2.71 ; S D=$ 1.590) als die alternative $(M=3.15 ; S D=1.703)$ bewertet.

- $\quad$ Die klassische Frageform wurde als sachlicher $(M=2.22 ; S D=1.528)$ als die alternative $(M=3.51 ; S D=1.852)$ bewertet.

Die Effektstärken sind für die Dimensionen „unseriös-seriös“, „,interessiertoberflächlich“, „befremdlich-angenehm" und "sachlich-unsachlich“ als groß zu bewerten (alle part.Eta ${ }^{2}$ zwischen .15 und .33). Für die Dimensionen „engstirnig-weltoffen“ (part.Eta $\left.{ }^{2}=.054\right)$ und "realitätsnahrealitätsfern" (part.Eta $\left.{ }^{2}=.044\right)$ zeigten sich eine mittlere bzw. kleine Effektstärke.

Auf das mit einem Freitext zu beantwortende Item „Die Fragen wirken außerdem:" wurde nur gelegentlich geantwortet. Mangels einer genügenden Anzahl von Antworten fand hier keine Analyse der wenigen Kommentierungen statt.

\subsection{Interpretation}

Aus Perspektive der ARqE muss hervorgehoben werden - hier durch den Dreischritt Ergebnisse-Interpretation-Diskussion versucht -, dass die Ableitungen von Aussagen aus der Betrachtung von Daten Interpretationen sind. Gerade hier lokalisiert Teo $(2008,2010)$ das Einsetzen von epistemologischer Gewalt, wenn Interpretationen nicht als solche expliziert werden, sondern stattdessen als neutrale Ableitungen dargestellt sind, auch wenn sie bestimmten Setzungen (z. B. was als normal gilt oder wer als erklärungsbedürftig begriffen wird) folgen. Daher wird hier nach der Darstellung der quantitativen Ergebnisse der Abschnitt Interpretation von einer Diskussion getrennt. Insgesamt wird darauf zu achten versucht, ein temporäres und lokales Phänomen zu beschreiben.

Es ließ sich mit dem eingesetzten Fragebogen bei der angesprochenen Stichprobe empirisch herstellen, dass die hier verwendete alternative Ge- 
schlechtsabfrage eine größere Geschlechtervielfalt bei der Selbstkategorisierung hervorrufen konnte.

Hier wird absichtlich die Formulierung mit „können“ verwendet, um zu betonen, dass dies ein temporäres und lokales Ereignis ist.

Weiterhin wirkte sich die Reihenfolge von verschiedenen Formen der Geschlechtsabfrage auf die Geschlechtervielfalt in den Antworten aus. Wurde zuerst auf einem binären Schema die geschlechtliche Selbstkategorisierung vorgenommen, dann wurde danach auf dem angebotenen Kontinuum von mehr Menschen eine Antwort gegeben, die das binäre Schema verlässt, als wenn die geschlechtliche Selbstkategorisierung zuerst auf dem Kontinuum vorgenommen wurde.

Geschlecht wurde im Kontext anderer, traditionell als relevant erachteter Personmerkmalen abgefragt. Da Geschlechterbinarität ein aktuell so dominantes Konstrukt ist, wurde der These nachgegangen, dass die alternative Geschlechtsabfrage unter den anderen alternativen Fragen als noch unseriöser als der gesamte alternative Fragenblock angegeben wird, bzw. weil die binäre Geschlechtsabfrage so herkömmlich ist, hätte sie als noch seriöser angegeben werden können, als der gesamte klassische Fragenblock. Dies ließ sich empirisch nicht herstellen, alle - deskriptiven und zudem sehr kleinen - unterschiedlich anmutenden Ergebnisse scheinen zur gewöhnlichen Varianz (Realisierungsraum) dieser Konfiguration zu gehören.

In einer klassischen Studie würde dies als zufällige Varianz bezeichnet werden.

Wenn Frageformen einen Unterschied auf den Bewertungsskalen hervorriefen, galt dies gleichermaßen für die Geschlechtsabfrage wie für den gesamten Frageblock. So wurde(n) die klassische(n) Frageform(en) als seriöser, oberflächlicher, angenehmer, weniger weltoffen, realitätsnäher und sachlicher als die alternative Frageform bewertet. 


\section{Form der Geschlechtsabfrage}

Die Konstellationen des Fragebogens in der benannten Gesellschaft am genannten Ort konnten eine geschlechtliche Selbstkategorisierung evozieren, die das klassische Binärschema weiblich-oder-männlich verließ. Obwohl Kenntnisse der Queer Theory in der befragten Stichprobe nicht erfragt wurden, gehe ich aufgrund des Ortes, an dem die Daten erhoben wurden, davon aus, dass diese Stichprobe als heteronormativ geprägt anzunehmen ist, wie dies für den Großteil der aktuellen europäischen Bevölkerung gilt. Dies würde bedeuten, dass auch ein substanzieller Anteil von heteronormativ geprägten Menschen - hier ca. die Hälfte der Studienpartner_innen - Freiheitsgrade bei der geschlechtlichen Selbstkategorisierung (die über ausschließlich weiblich oder männlich hinaus gehen) in Anspruch nehmen, wenn sie ihnen angeboten werden.

Interessant finde ich hierbei außerdem, dass beinahe alle Personen im jeweils vorgegebenen (binären oder nonbinären) Antwortschema bleiben. Nur eine Person hat die Linie des Kontinuums über „männlich“ hinaus verlängert und dort angekreuzt. Kein_e einzige_r Studienpartner_in hat beispielsweise das Binärschema verändert. Sie nutzten also nicht die Möglichkeiten, die ein Papierfragebogen bietet, um das Antwortformat zur Ausschöpfung von Freiheitsgraden zu verändern, sondern nutzten nur die Freiheitsgrade innerhalb des vorgegebenen Antwortschemas. Die Darstellung im Fragebogen schien zu befördern, dass jenen agential cuts, die durch die Gestalt der Fragen impliziert wurden, nachgekommen wurde. Dazu kommen Konfigurationen außerhalb des Fragebogens, wenn etwa Studienpartner_innen keine Fragebögen ungültig machen wollen oder auch andere Konfigurationen, die die Kooperation mit dem Frageformat fördern.

Ich schließe zunächst, dass es offensichtlich anderer als der hier gewählten Konfigurationen bedarf, um auch jene Freiheitsgrade der geschlechtlichen Selbstkategorisierung bzw. Non-Kategorisierung zu realisieren, in denen Studienpartner_innen ihr Antwortformat selbst generieren oder verändern. Solche Konfigurationen weist der als wissenschaftlich bezeichnete 
Fragebogen nicht auf. Gerade diese Konnotation der Wissenschaftlichkeit könnte eine diese-cuts-forcierende Konfiguration sein, während die Fragen eines (in unserer aktuellen Gesellschaft statusniedrigeren) Kindes sehr wahrscheinlich weniger wirksam wären. Hätte ein Kind das Kontinuum gezeichnet und würde die entsprechende Frage stellen, würden womöglich mehr Menschen dieser Zeit und diesen Ortes ungebundener mit dem Antwortformat umgehen. Eine solche Realisierung könnte in zukünftigen Studien versucht werden herzustellen. Im Moment möchte ich festhalten, dass das direkte Anbieten von Alternativen bezüglich geschlechtlicher Selbstkategorisierung deren Realisierung wahrscheinlicher zu machen scheint, als die herkömmlichen Intra-aktionen ohne das Anbieten.

An dieser Stelle wird durchaus der Geltungsbereich als ein wenig über den Rahmen des konkreten Fragebogens hinausgehend angenommen. Es bleibt offen, welche anderen Formen von Anbieten von Alternativen außerdem eine nonbinäre geschlechtliche Selbstkategorisierung wahrscheinlicher machen. Die Erörterung von möglichen Bedeutungen dieser Realisierung wird in der Diskussion fortgeführt.

Im Vergleich zur klassischen binären Abfrage wurden bei der alternativen Abfrage zwei Aspekte verändert: die Antwortmöglichkeit und die Frageformulierung. Es wäre möglich, in einer anderen Studie zu prüfen, ob sich auch dieses Ergebnismuster realisieren lässt, wenn ausschließlich die Antwortmöglichkeit verändert wird, also auf die Abfrage „Ihr Geschlecht:" das Kontinuum folgt. So wäre eventuell zu spezifizieren, ob die alternative Antwortmöglichkeit allein diese cuts zur Nonbinaritäts-Herstellung bereitstellt oder die Kombination aus der angewendeten Frageformulierung mit Antwortmöglichkeit.

\section{Form der Geschlechtsabfrage und Reihenfolge}

Die Konstellationen des Fragebogens riefen außerdem hervor, dass die geschlechtliche Selbstkategorisierung auf einem Kontinuum bzw. daneben („weder noch“-Option) von der Reihenfolge der Fragen abhängt, die Studienpartner_innen beantworten. 
Aus ARqE-Perspektive wird hier nicht spekuliert, ob sich in Abhängigkeit von der Reihenfolge auch die empfundene Selbstkategorisierung verändert oder nur die Antwort davon beeinflusst wird. In klassischer Denkweise könnte angenommen werden, dass Studienpartner_innen eine bestimmte Einschätzung ihrer Weiblichkeit/ Männlichkeit parat hätten, sich aber nicht trauen, diese zu äußern, wenn sie von der klassischen Binarität abweicht, solange sie nicht zuvor ankreuzen konnten, mit welchem Geschlecht sie gesellschaftlich gelesen werden. Aus ARqE-Perspektive kann immer nur über die erfolgte Selbstkategorisierung (und sei es auf Papier) gesprochen werden, denn diese hat sich - bei jenen, die eine Antwort gaben - realisiert. Von einer sogenannten inneren Einschätzung können wir bislang nur Unbestimmtheit annehmen.

Die Wahl von geschlechtervielfältigen Antworten auf die alternativ gestellte Geschlechtsabfrage wurde häufiger, wenn zuvor die klassischen Fragen beantwortet wurden. Es bleibt ungeklärt, welche genaue Konfiguration dies beeinflusst haben mag, denn es wurden ja ganze Fragenblöcke in der Reihenfolge getauscht. Es könnte also in einer nächsten Studie empirisch noch ausprobiert werden, dass geschlechtervielfältige Antworten auf die alternativ gestellte Geschlechtsabfrage wahrscheinlicher werden, wenn zuvor die Altersfrage klassisch gestellt wurde. Für eine solche cutErwirkung habe ich jedoch keine plausible Erklärung. Als etwas plausibler könnten jedoch folgende Überlegungen bewertet werden:

Autor_inn_en zahlreicher Veröffentlichungen - sowohl aus der akademischen Psychologie wie auch der Queer Theory - erwähnen oder beschreiben, wie umfassend und dominant das Konzept der Zweigeschlechtlichkeit in unserer Kultur ist (vgl. Butler, 1990/2006; Ebert und Steffens, 2013; Hegarty und Buechel, 2006; Perko, 2005). Viele kulturelle Intra-aktionen werden auch während Beantwortung des Fragebogens weiterhin in Richtung einer Herstellung von Geschlechterbinarität wirken. Nur bei explizitem Bezug zu beispielsweise Geschlechtsrolle (oder soziales oder psychologisches Geschlecht) wird schon seit den 1970er Jahren Varianz zu ausschließlicher Binarität diskutiert (vgl. Bem, 1974; Balzer Carr, Ben Hagai \& Zurbriggen, 2015). Also scheinen die kulturellen Normen bezüglich eines sogenannten 
sozialen Geschlechtes weniger stark in Richtung binärer Antworten zu beschneiden, als dies bei sogenanntem biologischen Geschlecht der Fall zu sein scheint. Wenn der Fragebogen nahelegt, dass es sich um soziales Geschlecht handelt, könnten möglicherweise eher geschlechterdiverse Antworten produziert werden, als wenn biologisches Geschlecht nahegelegt wird. Weiterhin kann es sein, dass die alternative Geschlechtsabfrageform eher als Frage nach sozialem Geschlecht verstanden wird, wenn sie als zweites gestellt wird und zuvor das sogenannte biologische Geschlecht angegeben werden konnte, als wenn sie die erste Frage im Fragebogen ist. Dies könnte eine Rolle dabei spielen, dass in der Reihenfolge erst klassisch, dann alternativ nach Angabe von Geschlechterbinarität auf die klassische Frage die Antworten auf die alternative Frage mehr Geschlechtervielfalt zeigten als in der anderen Reihenfolge (alternativ-klassisch).

Passend zur ARqE-Perspektive werden kontextuelle Zusammenwirkungen und nicht sogenannte intraindividuelle Verarbeitungsmechanismen in den Vordergrund gerückt. Dies schließt jedoch nicht aus, dass es Letztere als Realisierung situativ geben kann. Eine klassisch experimentalpsychologische Überlegung würde eher intrapersonale Erklärungen anführen, wie z. B. dass es den Studienpartner_inne_n schwerer fallen könnte, sich nonbinär zu kategorisieren, wenn sie vielfach gelernt haben, dass sie Frau oder Mann seien. Allerdings lokalisieren solche Erklärungen die Begründung vorrangig im Individuum und weniger im Kontext und sind deshalb aus ARqE-Perspektive zu minimieren. Auch implizieren Begründugen wie schwer fallen, dass es sich um eine Verzerrung von einem Eigentlichen handeln würde. ARqE würde dagegen argumentieren, dass es plausibel ist, dass sich Studienpartner_innen nach so massiver kultureller Binarisierung selbst binarisieren, und es interessant ist festzustellen, dass kleine Änderungen des Kontexts (hier z. B. das Frage- und Antwortformat) mehr Geschlechtervielfalt in einer Antwort hervorrufen können. ARqE fokussiert situiert auf den Kontext, solange die menschlichen Realisierungsmöglichkeiten Freiheitsgrade haben.

Unabhängig von der genauen Konfiguration - z. B. ob die Denkfigur verfügbar sein muss, dass ein biologisches Geschlecht von einem sozialen Geschlecht zu trennen wäre - führt das Ergebnis, dass die Reihenfolge 
mitgestaltet, die mögliche Flexibilität der geschlechtlichen Selbstkategorisierung in einer aktuellen heteronomativen Kultur vor. Dass die Reihenfolge eine Rolle spielt ist speziell bei der Frage "Wie fühlten Sie sich in den letzten Jahren meistens?" interessant. Wenn die Antwort darauf das Gefühl der letzten Jahre wiedergeben würde, dürfte sie sich nicht in Abhängigkeit von der Reihenfolge der Fragen ändern.

Aus klassisch experimentalpsychologischer Perspektive würde die Abhängigkeit der Antwort auf die Frage nach einem vergangenen Gefühl von der Reihenfolge der Fragen die Reliabilität oder Validität der betreffenden Messung in Frage stellen. Verständlicherweise könnte ein vergangenes Gefühl nicht durch die vorliegende Fragen-Reihenfolge verändert werden und wenn das Ergebnis sich abängig zeigt, stimme etwas mit der Messung nicht.

Wie genau die Intra-aktionen hervorrufende Konfiguration aussieht, die Geschlechtervielfalt in diesem Antwortformat hervorruft, bleibt zunächst unklar. Das Antwortmuster passt jedoch zu der Beschreibung von geschlechtlicher Selbstkategorisierung als Ergebnis von Handlungen (vgl. doing gender; West \& Zimmerman, 1987, Kessler \& McKenna, 1978, 2006) in Intra-aktion mit örtlichem Kontext (vgl. Barad, 2007): Geschlechtliche Selbstkategorisierung wäre demnach nicht inhärent in einer Person vorhanden, sondern würde situativ neu gebildet, basierend auf den realisierten Konfigurationen des mitbewirkenden Apparates.

\section{Bewertung der Fragen}

In der vorliegenden Studie ließ sich realisieren, dass klassische Formulierung im Vergleich zu alternativer Formulierung als seriöser, angenehmer, realitätsnäher und sachlicher angegeben wird, wenn mit so bezeichneten Likert-Skalen danach gefragt wird. Klassische Formulierung wurde außerdem als weniger weltoffen und eher als oberflächlich und die alternative eher als interessiert markiert. Auf diese Realisierung wirkte sich die Reihenfolge nicht aus. 
Welche genauen Intra-aktionen auch darauf hinwirken, dass die alternativen Fragen als weniger seriös etc. markiert wurden - diese sind offenbar so einflussreich, dass die cut-bewirkende Konfiguration der Reihenfolge an dieser Stelle keinen beobachtbaren Unterschied erzeugen konnte. Die Studie liefert keine Anhaltspunkte, ob die spezifischeren Thesen des zweiten Schrittes - beispielsweise, ob es wirklich an der Heteronormativität der bezeichneten Kultur liegt, dass die klassische Frageform als seriöser beurteilt wurde - als passend zu bezeichnen sind. Im Moment ist lediglich festzuhalten, dass sich unterschiedliche Bewertungen der verschiedenen Frageformen aufzeichnen lassen. Solche von Döring (2013) vermuteten Äußerungen von Einstellungen sind also realisierbar, obwohl bisher aus ARqE-Perspektive unklar ist, ob diese Aussagen erst durch die Fragen nach Einstellungen generiert wurden oder tatsächlich - auch vor der Frage danach - durch die Konfrontation mit der alternativen Frageform als (situierte) Einstellungen der Studienpartner_innen.

\subsection{Diskussion}

Der Dreischritt Ergebnisse - Interpretation - Diskussion soll salient machen, dass es nicht selbstverständlich ist, wie welches Datenmuster interpretiert wird, sondern dass die Interpretation vielmehr ein eigener Schritt der Auslegung ist. Auch in klassischen Studienbeschreibungen wird in der Diskussion eine inhaltliche Ableitung aus einem Datenmuster üblicherweise durch Betrachtung verschiedener Ableitungsmöglichkeiten relativiert. In ARqE-Studien sollte jedoch besonders viel Raum für solcherlei Nennungen sein, da die betrachtende Perspektive besonders relevant für die formulierten Aussagen ist. Es kann weiterhin Sinn machen, Interpretationen, die von den Vorannahmen abhängen, von der weiteren Bedeutungsdiskussion zu trennen bzw. dies zumindest zu versuchen. 


\section{Bedeutung der Form der Geschlechtsabfrage}

Dass der verwendete Fragebogen unter Passant_inn_en in einer deutschen Großstadt geschlechtliche Selbstkategorisierung hervorrufen kann, die nicht zum klassischen Geschlechterbinaritätskonzept passt, ist deshalb interessant, weil von den Studienpartner_innen keine besondere nonbinäre Prägung anzunehmen ist (von queertheoretisch beeinflussten Personen würde ich eher nicht-binäre Antworten erwarten). Die Daten passen zu der Beschreibung, dass Form und Kontext einer Frage mitgestalten, wie die Antwort auf diese Frage aussieht. Dabei kann sowohl ein größerer Kontext wie „Was ist in einer Kultur intelligibel?“, als auch die kleinschrittige Frageformulierung agential cuts bewirken. Eine bestimmte Frage kann zu einer anderen Zeit und an einem anderen Ort anders beantwortet werden als heute an diesem Ort. Eine Frage nach Geschlecht kann in Version A formuliert anders beantwortet werden als in Version B. Dies wird weder als Verzerrung noch als Messfehler gewertet, sondern vielmehr als Eigenart des Phänomens der geschlechtlichen Selbstkategorisierung. Ich verstehe individuelle geschlechtliche Selbstkategorisierung als ein heute und hier so lange unbestimmtes Phänomen, bis Konfigurationen eine situierte Realisierung aus den Potentialitäten Möglichkeits- (überhaupt nach Geschlecht zu fragen) und Realisierungsraum (zum Beispiel auf der alternativ formulierten Frageform) herausschneiden. Gleichzeitig rege ich weitere Studien aus ARqE-Perspektive an, die die wirkenden Konfigurationen für bestimmte Realisierungen erproben. Auch wenn wir Geschlecht als doing und nicht als being verstehen, ist interessant, welche Intra-aktionen zu welchem Resultat führen (können).

Im Prinzip kann man aufgrund des mangelnden Entitäten-Realismus im Agential Realism alles als doing-und-nicht-being beschreiben, auch wenn es kompliziert ist, nachzuvollziehen, dass verschiedenste Intra-aktionen eine situierte Realisierung herstellen und diese selbst Teil von Konfigurationen ist, welche wiederum eine weitere Realisierung mitbedingen. So entlarven geschlechtertheoretische Analysen die Denkfigur Geschlechterbinarität in unserer aktuellen Kultur als eine Realisierung, die selbst nicht stabil ist, sondern deren Stabilität uns 
durch Wiederholungen so erscheint und gleichzeitig dieses doing (Geschlechterbinarität) selbst cut-hervorrufendes situiertes being sein kann.

Interessant finde ich, wie scheinbar klein die Änderungen im lokalen und temporären Kontext waren und dennoch Änderungen im Phänomen der geschlechtlichen Selbstkategorisierung hervorrufen konnten. Die neben dem Fragebogen wirkenden Konfigurationen scheinen - trotz der andernorts schon herausgearbeiteten Wirkmächtigkeit der Heteronormativität, die nur Geschlechterbinarität zulasst - nicht so mächtig zu sein, dass nicht auch der Fragebogen mit seinen Eigenarten (Frageart und Reihenfolge) Einfluss ausüben kann. Ich plädiere dafür, die Persistenz einer geschlechtlichen Selbstkategorisierung nicht zu überschätzen. Eine AR-These könnte sein, dass wirkmächtige Intra-aktionen bei 99 von 100 Personen eine geschlechtliche Selbstkategorisierung realisieren, die als in den Personen und über deren Lebensspanne stabil erscheint, weil (situiert) keine mitwirkende Intra-aktion möglich ist, die das Ergebnis anders herausschneidet. Würden wir solche Phänomene finden, dann könnte man möglicherweise von einer gewissen Persistenz sprechen, auch wenn wir grundsätzlich annehmen, dass die Realisierung immer wieder neu hergestellt wird und nicht per se in der Welt existiert. Eventuell wäre die gravitative Anziehung von zwei physischen Körpern ein solches Beispiel, weil bisher keine Konfiguration bekannt ist, unter der dieses Ergebnis nicht stabil erscheint. Bezüglich psychologischer Phänomene halte ich eine solche Persistenz jedoch für höchst unwahrscheinlich. Bezüglich geschlechtlicher Selbstkategorisierung wäre in meiner Studie vorgeführt, dass allein die Fragenreihenfolge eine mitwirkende Intra-aktion darstellen kann.

Ich halte deshalb eine Auseinandersetzung mit verschiedensten aktuellen Einsatzpunkten von geschlechtlicher Selbstkategorisierung für lohnenswert. Allein bei einem Blick auf die oben genannten vielfältigen Aufforderungen zur Selbstkategorisierung - private Situationen, Personalausweis, Krankenakte, Versicherungsvertrag oder wissenschaftliche Fragebögen wird deutlich, dass einige Zusammenwirkungen und Konsequenzen unter der Annahme geringer Persistenz neu betrachtet werden müssen. Bei- 
spielsweise ist dann anzunehmen, dass nicht nur Personen, die sich selbst als weiblich kategorisieren (in Zusammenwirkung mit weiteren wirkenden Konfigurationen) andere Schönheitshandlungen vollziehen als Personen, die sich selbst als männlich kategorisieren, sondern dass dies auch für die Personen gilt, die sich selbst situativ auf dem Kontiuum als eher weiblich markieren etc. In Folge muss je nach Einsatzbereich, in welchem geschlechtliche Selbstkategorisierung realisiert wird, neben der gesellschaftlichen auch die wissenschaftliche Praktikabilität und Notwendigkeit beachtet werden. In welchen (sozial-)wissenschaftlichen Argumentationen spielt geschlechtliche Selbstkategorisierung eine Rolle und verändert sich jene, wenn die passendere Beschreibung dieses Phänomens wäre, wenig persistent und sehr kontextabhängig zu sein? Betrachten wir beispielsweise die schon erwähnte (sozial-) wissenschaftliche Betrachtung, wie geschlechtliche Selbstkategorisierung mit dem Umgang mit als geschlechtsuntypisch kategorisierten Gleichaltrigen zusammenhängt. Pauletti et al. (2014) halten zunächst fest (weil es sich empirisch zeigen lässt) dass Kinder, die äußern, geschlechtliche Unterscheidung zu favorisieren, sich selbst als geschlechtsuntypisch erachten und kein großes Selbstvertrauen angeben, sich über die Zeit hinweg aggressiver gegenüber als geschlechtsuntypisch kategorisierten Gleichaltrigen zeigen. Wenn wir nun aber davon ausgehen müssten, dass die geschlechtliche Selbstkategorisierung sehr kontextabhängig ist, so verändert sich die Bedeutung dieses Befundes. Es ist zwar möglich, dass auch die Intra-aktionen, die Pauletti et al. (2014) diskutieren, immer wieder realisiert werden können. Dies ist jedoch dann nur eine mögliche Beschreibung von Realisierungen. Welche Realisierungen sind noch möglich, wenn geschlechtliche Selbstkategorisierung in dem von Pauletti et al. (2014) beschriebenen Kontext viel variabler ist, als diese annehmen? Welche Realisierungen sind noch möglich, wenn ihre anderen Konzepte - wie z. B. Selbstvertrauen - ebenfalls keine große Persistenz aufweisen würden? Dies sind Fragen, die sich alle Fragebögen stellen müssen, die scheinbar nicht ohne geschlechtliche Selbstkategorisierung auskommen (vgl. Döring, 2013): Welche Realisierungen sind noch möglich? 
Da der Interessensfokus einer ARqE nicht darauf liegt, was ist, sondern darauf, was funktioniert, muss je nach Aussage über einen Sachverhalt klar sein, was situativ unter funktionieren verstanden wird. Erst dann können Realisierungen beschrieben werden, die verwendet werden können - ohne dass dies die einzig möglichen sein müssen. So könnten die Beschreibungen von Pauletti et al. (2014) verwendet werden, um in weiteren Konfigurationen aggressives Verhalten zu vermindern (dies wäre dann das Funktionieren und Verwenden). Möglicherweise eignen sich jedoch andere Beschreibungen besser. Weiterhin sollten Konzepte wie Selbstvertrauen, geschlechtliche Selbstkategorisierung und Geschlechtsuntypikalität nicht als inhärente stabile Eigenschaftsrealisierungen erachtet werden, bevor nicht ihre Persistenz untersucht und diskutiert wurde.

Interessant könnte eine Analyse sein, wieviele Nutzer_innen der Internetplattform Facebook nach der Bereitstellung von deutlich mehr als zwei Selbstkategorisierungsmöglichkeiten im Jahr 2014 ihre Geschlechtsangabe auf ihrer Profilseite umstellten und von einer vorher binären Kategorie zu einer nonbinären wechselten. Diese Wechsel könnten als Realisierung neuer Selbstkategorisierung nach Veränderung der Messkonfigurationen gelesen werden.

\section{Bedeutung der Form der Geschlechtsabfrage und Reihenfolge}

Dass die Reihenfolge einen Unterschied in der geschlechtlichen Selbstkategorisierung hervorrufen konnte, ist für folgende Überlegungen interessant. Einige psycholgische Arbeiten diskutieren die verschiedensten Zusammenhänge zwischen sogenannter Geschlechtsidentität und weiteren Variablen, wie z. B. der Schulfach-, Studien- oder Berufswahl. So wollen beispielseise Kessels, Heyder, Martin und Hannover (2014) eine Erklärung beisteuern, wie Schüler_innen auf lebenslang geschlechtlich gefärbte (Berufs-)Wege gelenkt werden. Sie verwenden für ihre Argumentation ein Verständnis von geschlechtlicher Selbstkategorisierung als stabil. Entsprechend empfehlen sie, dass Interventionen gegen einseitigen Fahrbahnen auf die mangelnde Passung zwischen sogenannter Geschlechtsidentität und geschlechtsstereotypen schulischen Entscheidungen abzielen sollten. Die 
hier vorliegende Studie könnte dafür sprechen auszuprobieren, ob sich schulische Entscheidungen durch Fragebogenkonfigurationen steuern ließen, die eine bestimmte geschlechtliche Selbstkategorisierung begünstigen. ${ }^{79}$ Sollte sich das realisieren lassen (sollte es also funktionieren), dann könnte eine Intervention sehr vereinfacht so aussehen: Vor der Wahl der Kurse für die Oberstufe in der Schule beantworten Schüler_innen Fragen, welche möglichst eine mittlere geschlechtliche Selbstkategorisierung herstellen.

Dies ist ein weiteres Beispiel für ein Phänomen, das sich empirisch zunächst in einer bestimmten Weise zeigen lässt, für das $\mathrm{ARqE}$ jedoch eine andere Interpretation anbietet, welche wiederum andere Folgeexperimente (z. B. ob die Fragebogenkonstellation eine Schulfächerwahl mitkonstruieren kann) und andere Folgehypothesen von Forschenden (z. B. darüber, wie eine Intervention aussehen kann) evoziert. Auch an einer solchen Stelle könnte es sein, dass Theoretiker_innen mit klassischer Perspektive argumentieren würden, dass die hypothetisierte Intervention eine unzulässige Beeinflussung von Schüler_innen darstellen würde. Jedoch aus der Perspektive, dass sogenannte Geschlechtsidentität etwas ist, das lokal und temporär realisiert wird, wäre auch die Wiederholung von Schülerinnen als weiblich eine ggf. unzulässige Beeinflussung. Diese Frage der Zulässigkeit muss dann auf anderer Ebene beantwortet werden - nämlich auf der Ebene „Welche Welt wollen wir?“ (vgl. auch Kapitel 4.2.5 zur Verantwortung von Forschenden und konsequenterweise auch von pädagogisch Handelnden)

\section{Bedeutung der Bewertung der Fragen}

Dass Studienpartner_innen die alternativen Fragen auf den gegebenen Skalen tatsächlich - wie von Döring (2014) spekuliert - als weniger seriös etc. markieren, ist meines Erachtens nicht per se, sondern nur im Hinblick auf weitere Zusammenhänge interessant. Dies gilt besonders, weil in der

\footnotetext{
79 Sehr wahrscheinlich wäre das keine Idee von Kessels et al. (2014), eben weil jene von einer stabilen Geschlechtsidentität ausgehen. Hier wird ihre Idee, dass die mangelnde Passung problematisch (weil einschränkend in der Wahl von Handlungen) ist, mit der Herangehensweise an Geschlechtsidentität als zunächst unbestimmt verknüpft.
} 
aktuellen Betrachtung offen gelassen werden muss, ob diese Bewertungen als eine Art innere Evaluation, unabhängig von Ihrer Abfrage, gesehen werden können, oder als erst durch die Abfrage hervorgerufene Bewertungen. Wenn beispielsweise Als-weniger-seriös-Markieren eher dazu führen würde, keine weiteren Fragen der Studie zu beantworten - was dem Wunsch der Forschenden entgegen stünde -, dann ist dies insofern interessant, als dass die Forschenden damit umgehen müssen. Wenn es jedoch keinen weiteren Zusammenhang - oder anders ausgedrückt, keine ersichtliche Folge dieser Art von Markierung - gibt, als dass Studienpartner_innen auf Nachfrage angeben, die alternativen Fragen als weniger seriös etc. zu empfinden, so ist diese Aussage zunächst bedeutungslos.

Wieder ist mit ARqE-Grundannahmen ein Ergebnis nicht per se interesant - vor allem nicht, weil Persistenz und Umfang der Lokalität eines Zusammenhangs bis jetzt unklar sind. Das Ergebnis wäre interessant, wenn es großen Konsens gäbe, dass das Beschriebene für viele Menschen zutrifft (im Sinne von immer wieder neu hergestellt wird) und mit großer Wahrscheinlichkeit auch über längere Zeit (so wie die gravitative Anziehung). Ansonsten ist es ein lokales und temporäres Ergebnis und es bleibt für eine Gesellschaft zu diskutieren, wie klein ein Wirkungsbereich sein darf bzw. wie groß er sein muss, damit Aussagen da-rüber als interessant gelten.

\section{Kritik der Studie}

Gerade auch in einer Studie, die aus Perspektive der ARqE durchgeführt wird, sollte sich ein Abschnitt der Diskussion selbstkritisch mit der Studie, ihren Voraussetzungen und möglichen Wirkungen auseinandersetzen. Dies soll keine Doppelung zu den stets begleitenden kritischen Reflexionen von cut-Setzungen durch die Studienkonfigurationen (wie beispielsweise, dass Bewertungen möglicherweise allein durch die Frage danach hergestellt werden) sein, sondern zusätzliche Problemstellen thematisieren bzw. Problemstellen zusammenfassen und ihre Problemhaftigkeit einordnen.

Beispielsweise sollte sich eine ARqE-Studie der Frage widmen, welche Art von regulatorischen Operationen die Reifizierung des Konzeptes Geschlecht 
überhaupt beinhaltet und wie diese regulatorischen Operationen zu bewerten sind. In der vorliegenden Studie wurde die momentane Häufigkeit und Relevanz des Konzeptes an bezeichnetem Ort und zu bezeichneter Zeit als Grund angeführt, das Konzept zu wiederholen und damit zu reifizieren. Allgemein sollten Studien sich umfassend damit beschäftigen, welche alternativen Realisierungen möglich sind, als Menschen in Geschlechtskategorien einzuteilen, und weiterhin, aus welchen Gründen welche Realisierungen wünschenswert für eine Gesellschaft sein können.

In der Reflektion der möglichen Wirkung der Studie auf Studienpartner_innen muss erstens die Wiederholung des Konzeptes Geschlecht bedacht werden. Mit ihrem ganzen Aufbau wiederholt und stabilisiert die Studie das Konzept Geschlecht. Zwar bietet sie allen Studienpartner_innen explizit die Möglichkeit, sich außerhalb der gängigen zwei Kategorien einzugruppieren, indem Studienpartner_innen entweder etwas dazwischen oder die Option „weder noch“ wählen können. Dennoch fragt sie alle Studienpartner_innen direkt - und sogar zweimal - nach geschlechtlicher Selbstkategorisierung. Insgesamt wird das Phänomen der geschlechtlichen Selbstkategorisierung für die konkreten Studienpartner_innen damit womöglich eher stabilisiert statt komplett kritisiert. Dies ist queertheoretisch problematisch und aus meiner Sicht nur akzeptabel, weil das Konzept Geschlecht aktuell in der angesprochenen Population sehr wahrscheinlich sehr alltäglich ist (vgl. Butler, 1990/2006; Perko, 2005; Warner, 1991). Eine solche Wiederholung würde ich dann für so problematisch halten, dass die Studie hätte unterlassen werden müssen, wenn eine Population angesprochen werden sollte, für die das Konzept Geschlecht (noch) nicht (so) selbstverständlich ist. Letzteres würde ich z. B. für Kinder oder Personen, die das Konzept Geschlecht ablehnen, annehmen. Erstere wurden aus diesem Grund von der Teilnahme ausgeschlossen, Letztere kommen auch in unserer aktuellen heteronormativen Gesellschaft vor und damit möglicherweise auch in der berichteten Stichprobe. Diese würden von dem verwendeten Fragebogen erneut zur geschlechtlichen Selbstkategorisierung angehalten, was aus queertheoretischer Sicht als an der Studie problematisch zu bewerten ist. 
Aus queertheoretischer Sicht wünschenswerterweise kann die klassische Geschlechterbinarität immerhin destabilisiert werden. Jeder Studienpartnerin_jedem Studienpartner wurde bei der Beantwortung der alternativen Geschlechtsabfrage zumindest angeboten, neben „weiblich“ und „männlich“ alternative Antwortmöglichkeiten in Betracht zu ziehen. Ein_e Studienpartner_in antwortete beispielsweise auf die klassische Binärfrage mit „weiblich“ und kreuzte auf dem Kontinuum zweimal „männlich“ an. Damit bewegten sich ihre_seine Kontinuumantworten weiterhin im Binärschema (und wurden auch als binär gezählt). Sie bewegen sich jedoch außerhalb der heteronormativen Annahme, dass Personen konstant dasselbe Geschlecht angeben sollten bzw. gesunde Menschen dies tun. Ich verstehe dies als Bekräftigung der Beschreibung, dass Menschen bei dieser alternativen Geschlechtsabfrage zumindest über Alternativen zu „weiblich“ und „männlich“ nachdenken. Insgesamt führte die Studienkonstellation dazu, dass Studienpartner_innen sich auch nonbinär geschlechtlich kategorisierten. Daraus schließe ich, dass die verwendeten Frageformen für Studienpartner_innen zwar das Konzept Geschlecht reifizierten, jedoch das Konzept Geschlechterbinarität destabilisierten. Sollte darüberhinaus die Rezeption solcher Studien bei anderen Forschenden das Konzept Geschlecht destablisieren, wäre dies eine weitere queertheoretisch wünschenswerte Wirkung.

Diese Beispielstudie weist also auch aus queertheoretischer Sicht problematische Umsetzungen auf. Dies soll jedoch verdeutlichen, dass einem Abwägungsprozess nicht $\mathrm{zu}$ entkommen ist und ARqE-informierte Ausarbeitungen darüber, was als wünschenswert gelten kann, noch ausstehen.

Aus ARqE-Perspektive ist weiterhin zu fragen, ob die Verwendung von geschlechtlichen Binärkategorien bei der Abfrage einer geschlechtlichen Selbstkategorisierung nicht den regulatorischen Operationen von Butler entspricht, was zu vermeiden wäre.

Butler benennt jene „restriktiven Diskurse“ als regulatorische Operationen, die darauf bestehen, dass es ausschließlich Frau und Mann als intelligible Möglichkeiten gibt: 
Thus, a restrictive discourse on gender that insists on the binary of man and woman as the exclusive way to understand the gender field performs a regulatory operation of power that naturalizes the hegemonic instance and forecloses the thinkability of its disruption. (Butler, 2004, S. 43)

Stellt also die Ausgestaltung des Fragebogens einen restriktiven Diskurs über Geschlecht dar, der nur Mann und Frau als verstehbar zulässt?

Die klassisch binär gestellte Geschlechtsabfrage ist tatsächlich eine regulatorische Operation, auch im Sinne einer Einschränkung, denn sie wiederholt die Suggestion, dass es auf die Frage nach dem Geschlecht als plausible Antworten nur „weiblich“ und „männlich“ gäbe. Sie reduziert die Antwortmöglichkeiten von Personen innerhalb einer gesellschaftlich noch als sehr relevant erachteten Bezeichnung und beteiligt sich an der Wiederholung heteronormativer Geschlechterbinarität. Allerdings wurde in der Studie explizit darauf geachtet, dass keine Person nur mit der binär gestellten Frage konfrontiert wurde. Alle Studienpartner_innen erhielten - wenn auch in unterschiedlicher zeitlicher Abfolge - zudem die alternative Frageund Antwortform. Der Fragebogen realisiert - trotz teilweiser Wiederholung der klassischen Form - ein Aufbrechen der reinen Binarität. In meinen Augen wiederholt der Fragebogen als Ganzes daher den restriktiven Diskurs nicht.

Sollte durch das Arrangement (Reihenfolge) und die Gestalt der Fragen im bestehenden Kontext (z. B. im Hinblick auf Konversationsnormen, die besagen, dass eine sinnvolle zweite Frage nicht dasselbe erfragt wie eine vorher gestellte) der Eindruck bei Studienpartner_innen evoziert worden sein, dass sogenanntes biologisches Geschlecht von sogenanntem sozialen Geschlecht zu trennen sei, würde der Fragebogen allerdings ein queertheoretisch zu kritisierendes Ergebnis fördern. Dies halte ich im Moment aber für nicht beurteilbar.

Neben der Verwendung von Geschlechtskonzeptionen wirft zudem die Realisierung der Bewertung von Fragen als "seriös" etc. gleich mehrere Probleme auf. Zwar findet sich eine in Abhängigkeit von der Frageart (klassisch 
oder alternativ gestellte Fragen) unterschiedliche Markierung der LikertSkalen. Das Ergebnis bewerte ich jedoch als lokal und temporär mit geringem Geltungsbereich und Geltungszeitraum. Es bleibt zudem unklar, ob dieses Ergebnis allein durch die Messung hergestellt wurde oder andere Intra-aktionen außer der Messung an der Realisierung beteiligt waren. Ein solcher Sachverhalt ist insgesamt ungünstig und für solcherlei Forschungsteile sollten aus ARqE-Perspektive keine Ressourcen eingesetzt werden.

Für die vorliegende Arbeit lohnen die eingesetzten Ressourcen, weil der Fokus auf die Erläuterung der ARqE-Perspektive gerichtet ist und es nicht primär darum geht, Effekt-Realisierungen vorzuführen. In einer rein inhaltlichen Studie wäre aus ARqE-Perspektive von einem Vorgehen abzuraten, das Bewertungen misst, ohne davon ausgehen zu können, dass mehr als die Messung alleine diese herstellt (vgl. Kapitel 4.2.3) bzw. ohne die Absicht, weitere Zusammenwirkungen zu beleuchten.

Insgesamt muss in einem Forschungsprozess, welcher von der Mitherstellung seiner Gegenstände ausgeht, ein neuer Umgang mit ethischen Entscheidungen entwickelt werden. Im Moment fehlen Standards dafür. Ebenfalls fehlt Forschung aus ARqE-Perspektive im inhaltlichen Bereich. Bei zahlreichen Phänomenen - allein in dieser Beispielstudie - ist bislang unklar, welche Intra-aktionen an der jeweiligen Realisierung beteiligt sind und in welchen Kontexten wir welche Ergebnisse materialisiert vorfinden. Beispielsweise können sich Eltern heutzutage in Deutschland die größte Mühe geben, ihr Kind nicht geschlechtlich zu kategorisieren, aber die Umwelt wird es dennoch beinahe allumfassend tun. Für andere Konzepte (wie z. B. Extraversion vs. Introversion) wirken dagegen vielleicht nicht jederzeit und allenorts Intra-aktionen, die diese herstellen.

Wenn ARqE-Forschung in relevanter Menge einmal vorliegen sollte, könnte eher ein Konsens gefunden werden, was alles zu spezifischen Realisierungen beiträgt. Konzepte wie Selbstvertrauen, geschlechtliche Selbstkategorisierung und Geschlechtsuntypikalität sollten in jedem Fall nicht per se als inhärente stabile Eigenschaftsrealisierungen erachtet werden, bevor nicht ihre 
Persistenz untersucht und diskutiert wurde. Alternative Realisierungsmöglichkeiten sollten empirisch erprobt werden. So lässt sich auch der Realisierungsversuch kontextabhängige geschlechtliche Selbstkategorisierung noch ausweiten und verbessern.

Die heteronormative Grundannahme über Geschlechterbinarität wird in der Studie nur teilweise aufgebrochen, da sie zu einem gewissen Teil auch wiederholt wird. Die heteronormative Grundannahme, dass Frauen „normalerweise" weiblich und Männer "normalerweise" männlich sind, wird nicht wiederholt. Der Fragebogen legt den Studienpartner_innen auch nirgends nahe, dass dies bessere Kombinationen seien als andere mögliche Kombinationen. Ebenso versucht der Fragebogen, die Antwort "weder noch" als gleichwertige, intelligible und gewöhnliche Antwortmöglichkeit anzubieten. Wenn weiterhin Intra-aktionen wirken, die diese Antwort unwahrscheinlicher machen, so ist dies m. E. wenig durch die Konfiguration des Fragebogens gegeben.

Wichtig ist, dass von keiner stabilen geschlechtlichen Identität ausgegangen wird. Gleichzeitig wird eine solche Konzeption auch nicht verhindert, so dass Studienpartner_innen problemlos für sich eine stabile geschlechtliche Identitätskonstruktion annehmen können - dies ist schlicht für die Studie irrelevant. Damit kann sie an dieser Stelle einen queertheoretisch positiv zu bewertenden Ort mit ungewöhnlichen Freiheitsgraden in Bezug auf individuelle Identitätskonstruktionen bieten. Die in der Studie fokussierte situierte Selbstkategorisierung interessiert unabhängig davon, ob sich diese individuell stabil oder veränderlich zeigt.

Bezüglich Intersektionalitätsansprüchen ist die Fremdkategorisierung nur bei der Anwerbung der Studienpartner_innen in Kinder versus Erwachsene relevant. Diese Fremdkategorisierung sollte durchkreuzt mit anderen Differenzachsen gesehen werden. In anderen Kulturkreisen würden eventuell die minderjährigen und ggf. auch noch älteren Studienpartner_innen nicht als Erwachsene verstanden oder andersherum die hier ausgeschlossenen Kinder als ungerechtfertigt ausgeschlossen betrachtet. 
Bezüglich Wirkung von Machtpositionen gebe ich Folgendes zu bedenken: Der Fragebogen wurde als Teil einer Doktorarbeit präsentiert. Dies hatte möglicherweise andere Wirkungen im Hinblick auf die enstandenen Antworten, als wenn er beispielsweise als von Schüler_innen stammend präsentiert worden wäre. Wenn die Präsentation als Doktorarbeit (überhaupt) zu Gedanken bei Studienpartner_innen führte, könnte dies die machtvolle Position von Wissenschaft stärken und trüge nicht zum Abbau dieser Hierarchie bei. Allerdings ist auch - in die Richtung, in die Döring (2013) vermutete - denkbar, dass diese Art der Fragen und ihre folgende Einschätzung als dubios das Bild der Wissenschaft verschlechterte. Wie genau solcherlei Fragebögen sich auf bestimmte Diskurse über Wissenschaft auswirken, kann ich anhand der Studie nicht sagen.

Im Verlauf der Fragebogenbeantwortung geben Studienpartner_innen mitunter als recht privat empfundene Informationen an für sie fremde Personen weiter. Auch wenn die Fragen anonym beantwortet wurden und jede Frage ausgelassen werden konnte, könnten aktuelle soziale Normen nahelegen, dass man eine einmal begonnene Beantwortung nicht (gleich) wieder abbrechen sollte - auch wenn man die Fragen mittlerweile lieber doch nicht beantworten würde. Eine Person brach die Beantwortung des Fragebogens mit der Begründung ab, dass die Fragen zu privat seien. Dies zeigt, dass die verwendeten Fragen als zu privat empfunden werden können. Forschende sind demnach meist schon deshalb in der mächtigeren Position, weil sie - anders als die Studienpartner_innen - keine persönlichen Informationen preisgeben sollen. Die klassische Art eines Fragebogens erhält dieses Machtgefälle statt es aufzubrechen.

Weiterhin sind aus machtkritischer Perspektive Interpretationen von Forschenden über Studienpartner_innen problematisch - vor allem wenn diese nicht mit den Studienpartner_innen rückgekoppelt wurden (beispielsweise Dialog-Konsens-Methoden beinhalten eine solche Rückkoppelung, siehe Scheele und Groeben, 1988). Die vorliegende Studie erprobt eine geschlechtervielfältige Realisierung und zielt nicht explizit darauf $a b$, Personen oder ihr Verhalten zu interpretieren. Dennoch kommen Interpretationen über Studienpartner_innen vor - etwa, wenn jene 
als höchstwahrscheinlich heteronormativ geprägt angenommen werden. Da diese Aussagen aber nicht Ergebnisse der Studie sind, beteiligt sich die Studie nicht vorrangig an einer Klassifikation von Personen. Intendiert war vielmehr, die Gleichbehandlung von aktuell kulturell typischer und untypischer geschlechtlicher Selbstkategorisierung und damit deren zukünftige kulturelle Gleichbereichtigung zu fördern.

Die in der Studie vorgeführte Realisierung von Geschlechtervielfalt, ist schon vor Besprechung der Studie - sprachlicher Natur, da nur Frage- und Antwort-Verhalten beachtet wurde, das sprachlich hergestellt ist. Sowohl der Ergebnis-mitbestimmende Apparat wie auch die resultierenden Realisierungen sind in diesem Fall sprachlich.

Abschließend möchte ich erwähen, dass die Studie eventuell Anregungen dahingehend liefern kann, in einer zukünftigen Auseinandersetzung bzw. ARqE-Studie das Alter als angeblich bedeutungsvolles inhärentes Merkmal von Menschen zu entselbstverständlichen. Wie eingangs spekuliert, kommt von Studienpartner_innen, die ihr Alter als 15 - 18 angeben, die Nennung vor, dass eine Beurteilung als Minder- oder Volljährig ja nur eine Außenkategorisierung ist. Es gibt also Situationen, in denen Menschen nicht ihr sogenanntes (als Merkmal verstandenes) Alter für ihre Erfahrungen verantwortlich machen, sondern den (gesellschaftlichen) Umgang damit. In dieser Studie wurde auf geschlechtliche Kategorisierung fokussiert und auf weitere Analysen bezüglich der Begründungen, warum Menschen ihr Alter (nicht) auffällt, verzichtet.

Insgesamt zeigt die Studie, dass bei Menschen, die an einem öffentlichen, zentralen Ort einer deutschen Großstadt einen Fragebogen ausfüllen, bei der Frage nach geschlechtlicher Selbstkategorisierung sowohl die Frageart als auch die Reihenfolge eine cut-produzierende Konfiguration sein kann, die die resultierende Realisierung mitbestimmt. Da eine vom Fragebogen angebotene Varianz bei der Selbstkategorisierung durchaus in Anspruch genommen wird, gleichzeitig aber ein vorgegebenes Binärschema weder verändert wird noch unbeantwortet bleibt, sollten zukünftige Fragen nach 
geschlechtlicher Selbstkategorisierung diese cut-Produktionen in ihren eigenen Designs beachten.

\subsection{Fazit der Erprobung}

Diese Auseinandersetzung mit einer Studie konnte nur exemplarisch einige Formulierungen und inhaltliche Punkte aufgreifen. Es wird deutlich, dass für die konkrete Gestaltung von (psychologischen) empirischen Arbeiten, die einen Agential Realism zugrunde legen und eine queertheoretische Perspektive einnehmen, noch zahlreiche Praktiken entwickelt werden müssen. Erste Schritte von Vorgehensweisen wurden hier dennoch aufgezeigt und diskutiert.

Heteronormativität wird insofern aufgebrochen, als das klassische Binärschema für geschlechtliche Selbstkategorisierung nicht nur wiederholt, sondern in der alternativen Abfrage auch neu gestaltet wird. Queerend ist dabei, dass jede_r Studienpartner_in auch die alternative Abfrage bearbeitete und nicht manche nur die klassische, Binarität reifizierende Abfrage erhielten. Heteronormativität stützend wäre dagegen, wenn die Gestalt der Fragebögen bei den Studienpartner_inne_n die Annahme evozierte, dass es nur bei sogenanntem sozialem Geschlecht sinnvolle und nicht-pathologische Varianz geben kann, aber nicht bei sogenanntem biologischem Geschlecht. Damit ist die vorliegende Studie mit ihrem Gehalt an Aufbrechen von Heteronormativität wohl nicht die stärkste Konfiguration, kann aber - so hoffe ich - dennoch die alternative Denkrichtung vorführen.

Queerend bezüglich Identitätskategorien ist die Studie insofern, als sie über Geschlecht - was mitunter als Ressource einer sehr starken und wichtigen Idenitätskategorie beschrieben wird - nicht als Identitätskategorie spricht. Die geschlechtliche Einordnung wird nur als situierte Selbstkategorisierung behandelt und an keiner Stelle als eine Fremdkategorisierung verwendet. Weiterhin wird auch den Lesenden, die geschlechtliche Selbstkategorisierung als identitäre 
Kategorisierung verstehen, zu verdeutlichen versucht, wie kontextabhängig das Ergebnis einer Kategorisierung ist. Damit schreibt die Studie - wie queertheoretisch gefordert - die Einordnung nicht fest, sondern zeigt deren Flexibilität und Situiertheit und lässt gleichzeitig Raum für individuelle Selbstdefinitionen der Personen.

Intersektional hätte eine soziale Kategorie betrachtet werden müssen, wenn die Studie über Mitglieder einer sogenannten Gruppierung gesprochen hätte. Das tut sie in einem Fall, da von außen in sogenannte Kinder und sogenannte Erwachsene unterschieden wurde. Diese Differenzierungsachse kann in anderen Settings anders verlaufen, wenn Personen, die in Deutschland als Kinder gelten, an anderen Orten als schon erwachsen verstanden werden, oder Personen, die in Deutschland als Erwachsene gelten, an anderen Orten als Kinder gelten. Wenn in der Studie über sogenannte Kinder gesprochen wird (um sie als Studienpartner_innen auszuschließen), dann wird gleichzeitig über ein heutiges, deutsches Verständnis von Kindsein gesprochen. Weitere aktive Fremdkategorisierungen werden in der Studie nicht vorgenommen, sondern es werden mitwirkende Konfigurationen der Herstellung thematisiert. Deshalb kann der Intersektionalitätsanspruch bezüglich Identitätskategorien in den Hintergrund treten. Dass alle Studienpartner_innen eine mit anderen Differenzierungsachsen verschränkte Vorstellung von weiblich etc. haben, ist grundsätzliche Logik des Vorgehens und für die hervorgebrachten Effekte irrelevant.

Im Vorgehen des Realisierungsversuchs und der Diskussion der miterwirkenden Intra-aktionen ist implementiert, dass Macht (im Foucault'schen Sinne) Phänomene hervorbringt und es keine Position außerhalb von wirkenden Machtverhältnissen gibt. Durch Zu-grundelegen der Logik des Agential Realism wird ständig reflektiert, dass in der Betrachtungssituation der Studie selbst quasi in ihrem Messvorgang - Machtrelationen wirken und gewisse Konzepte stabilisiert, andere destabilisiert werden. Die Sensibilität gegenüber machtungleichen Positio-nen zeigt die Studie beispielsweise bei der Diskussion von Interpretationen über Studienpartner_innen als heteronormativ geprägte Stichprobe. Insgesamt weist die Studie aber durch den Fokus auf das Erproben einer geschlechtervielfältigen Realisierung einen geringen Grad an externer Einord- 
nung von Studienpartner_innen auf und beteiligt sich dadurch nicht maßgeblich an der Stabilisierung von Wissenschaft als jene Instanz, die immer besser als Individuen Aussagen über Realitäten treffen könnte.

Bezüglich der Bedeutung von Sprache und Sprechakten kommt zum Tragen, dass die Antworten sprachlicher Natur sind. Auch wenn das Setzen eines Kreuzes auf einem Kontinuum eine nicht-sprachliche Komponente aufweist, halte ich die sprachliche Komponente für die weit bedeutsamere, da das Kontinuum erst durch die sprachliche Bezeichnung Bedeutung erhält. Die Bedeutung der Sprache für die Herstellung der geschlechtlichen Selbstkategorisierung wird hier nicht weiter diskutiert. Es soll jedoch klar kommuniziert werden, dass die erprobte Realisierung in diesem Fall sprachlicher Art ist.

Die queertheoretische Anforderung nach Beachtung der Historizität und Kontextabhängigkeit eines jeden Konzeptes wird durch Spezifizierungen des Ortes und der Zeit sowie durch das Design eingelöst, wobei letzteres die Kontextabhängigkeit geradezu vorführen will. Selbst für beispielsweise die Bewertung als un/seriös wird in Frage gestellt, ob diese zwangsläufig entsteht, da keinerlei Hinweise vorliegen, dass sie auch ohne ihre explizite Abfrage entstanden wäre. So wird geradezu auf die Spitze getrieben, dass eine Bewertung möglicherweise nur am Ort und zur Zeit der Fragebogenbeantwortung existiert, weil dieser Akt die Bewertung erst evoziert.

Was solche Studien alles dekonstruieren (können), wenn sie rezipiert und ernst genommen werden, ist eine offene Frage. Aus meiner queertheoretisch informierten Perspektive halte ich u. a. folgende Dekonstruktionen für besonders erstrebenswert: Dekonstruktion von Geschlechterbinarität, Dekonstruktion von Konstrukten als kontextlos gegeben, Dekonstruktion von Forschenden als per-se Wissenderen, Dekonstruktion von Wissen als ort- und zeitlose Faktenkenntnisse. Ich gehe davon aus, dass nicht etwa einzelne Studien diese Dekonstruktionen einzulösen vermögen, sondern eher Herangehensweisen wie queerfeministische Kritik, Situated Knowledges ${ }^{80}$ und Agential Realism dazu geeignet sind. Ziel

80 Im Sinne von Donna Haraway (beispielsweise 1988) 
dieser Arbeit ist es jedoch auch, mit ARqE eine Form empirischer Forschung vorzustellen, deren - erst noch durchzuführende - Studien in der Zusammenschau ebenfalls dekonstruierende Wirkung entfalten können.

Vorrangiges Ziel dieses Kapitels ist es, einen Eindruck über das Vorgehen von ARqE-Studien zu geben, und weniger, konkrete Aussagen über situiert wirkende Intra-aktionen im Sinne einer $\mathrm{ARqE}$ zu generieren. Auch wenn meines Erachtens ein erstes Vorführen gelungen ist, hätte ich aus der aktuellen Perspektive zwei Punkte des Studiendesigns anders gestalten können, um ein stärkeres Argument formulieren zu können.

Erstens wäre die Demonstration, dass die Formulierung des Messintrumentes den erzeugten Gegenstand mit herstellt ggf. noch überzeugender, wenn sich nur die nonbinäre Antwortformulierung - und nicht auch die Frageformulierung - von der klassisch binären Variante unterscheiden würde. In den oben getroffenen Entscheidungen hatte ich der Ähnlichkeit der beiden nonbinären Fragen untereinander Priorität eingeräumt (vgl. S. 207), statt der Ähnlichkeit der binären und nonbinären Abfrage. Auch wenn die Ähnlichkeit zwischen der binären und nonbinären Abfrage aus ARqE-Perspektive nicht nötig ist, würde sie mir nun auf dem Weg von einem klassischen hin zu einem ARqEinformierten Design als ein kleinschrittigerer Übergang erscheinen. Dafür müsste eine Formulierung der Geschlechtsabfrage gefunden werden, die für beide Antwortformate passt. Alternativ müsste riskiert werden, dass es aktuell an den umgrenzten Orten merkwürdig wirken kann, wenn eine Frage in identischer Form zweimal gestellt wird. Eine weitere Möglichkeit wäre, diese Bedingung doch interindividuell zu variieren. Dann müssten aus ARqE-Perspektive jene Personen, die ausschließlich die binäre Formulierung vorgelegt bekommen, ein differenziertes Debriefing erhalten, welches die reifizeirte Heteronormativität aufzubrechen versucht. Allerdings ist in diesem Zusammenhang interessant, welche Auswirkungen es hatte, dass die Internetplattform Facebook in 2014 die Möglichkeit einführte, Geschlecht nicht nur als entweder weiblich oder männlich, sondern als benutzerdefiniert anzugeben. Jede Person, die ihre Geschlechtsangabe in benutzerdefiniert geändert hat, wäre eine Demonstation der wirkenden Intra-aktion durch das Antwortformat, auch wenn die Frage identisch geblieben 
ist. Ich gehe zusammenfassend davon aus, dass für die geschlechtliche Selbstkategorisierung in unserer heutigen Kultur sowohl das Antwortformat alleine, wie auch Frageformat mit Antwortformat zusammen eine cut-erwirkende Intraaktion sein kann.

Zweitens hätte das von Döring verwendete Wort „dubios“ in der Herstellung der Bewertung der Fragen vorkommen sollen (vgl. Döring, 2013, die vermutete, nonbinäre Geschlechtsfragen könnten eine ganze Studie als „dubios“ erscheinen lassen). Ich sehe aus jetziger Perspektive keinen Grund, dieses Wort nicht genauso auszuprobieren wie die anderen auch. Im Gegenteil legt die Besprechung von Dörings These im Rahmen der Studie die Verwendung des Wortes „dubios“ geradezu nahe.

Abschließend möchte ich noch eine andere Auseinandersetzung als Erprobung der ARqE-Perspektive vorschlagen. In meinem Artikel "Queering Identities in Psychology: Blind Alleys and Avenues" (Scholz, 2016; in Queering Paradigms VI: Interventions, Ethics, and Glocalities) betrachte ich die psychologisch klassische Selbstkategorisierungstheorie von Turner, Hogg, Oakes, Reicher und Wetherell (1987) und welche Umformulierungen der Theorie aus queertheoretischer Sicht nötig wären. Ohne explizit den Agential Realism zu nennen, verwende ich darin eine Herangehensweise an Phänomene als situiert, an Realisierungen als non-essentiell sowie an Identitäten als lokal und temporär und expliziere die im Text ebenfalls gesetzen cuts, die selbst kontextabhängig sind. Dieser Artikel stellt keine Studie vor, sondern betrachtet eine psychologische Theorie sowie deren queertheoretisch adaptierbaren Anteile und diskutiert queertheoretisch wünschenswerte Neuerungen. Mit solchen Auseinandersetzungen möchte ich anregen, mit Science zu arbeiten und im Sinne von Barad und Butler keine human-independent facts about nature (vgl. Barad, 1996, S. 162) zu generieren, sondern intra-actively entangled ones (vgl. Barad, 2007).

Open Access Dieses Kapitel wird unter der Creative Commons Namensnennung 4.0 International Lizenz (http://creativecommons.org/licenses/by/4.0/deed.de) veröffentlicht, welche die Nutzung, Vervielfältigung, Bearbeitung, Verbreitung und Wiedergabe in jeglichem Medium und Format erlaubt, sofern Sie den/die ursprünglichen Autor(en) und die Quelle ordnungsgemäß nennen, einen Link zur Creative Commons Lizenz beifügen und angeben, ob Änderungen vorgenommen wurden.

Die in diesem Kapitel enthaltenen Bilder und sonstiges Drittmaterial unterliegen ebenfalls der genannten Creative Commons Lizenz, sofern sich aus der Abbildungslegende nichts anderes ergibt. Sofern das betreffende Material nicht unter der genannten Creative Commons Lizenz steht und die betreffende Handlung nicht nach gesetzlichen Vorschriften erlaubt ist, ist für die oben aufgeführten Weiterverwendungen des Materials die Einwilligung des jeweiligen Rechteinhabers einzuholen. 